\title{
Invisible decays of axion-like particles: constraints and prospects
}

\author{
Luc Darmé, ${ }^{a}$ Federica Giacchino, ${ }^{a}$ Enrico Nardi ${ }^{a}$ and Mauro Raggi ${ }^{b}$ \\ ${ }^{a}$ Laboratori Nazionali di Frascati, Istituto Nazionale di Fisica Nucleare, \\ via Enrico Fermi 54, 00044 Frascati, Italy \\ ${ }^{b}$ Dipartimento di Fisica, Università di Roma La Sapienza and INFN, Sezione di Roma, \\ Piazzale Aldo Moro 2, I-00185 Rome, Italy \\ E-mail: luc.darme@lnf.infn.it, fgiacchi@lnf.infn.it, \\ enrico.nardi@lnf.infn.it, mauro.raggi@roma1.infn.it
}

ABSTRACT: Axion-like particles (ALPs) can provide a portal to new states of a dark sector. We study the phenomenology of this portal when the ALP mainly decays invisibly, while its interaction with the standard model sector proceeds essentially via its coupling to electrons and/or photons. We reanalyse existing limits from various collider and beam dump experiments, including in particular ALP production via electron/positron interactions, in addition to the usual production through ALP-photon coupling. We further discuss the interplay between these limits and the intriguing possibility of explaining simultaneously the muon and electron magnetic moment anomalies. Finally, we illustrate the prospects of ALP searches at the LNF positron fixed-target experiment PADME, and the future reach of an upgraded experimental setup.

KeYwords: Beyond Standard Model, Thermal Field Theory

ArXiv ePrint: 2012.07894 


\section{Contents}

1 Introduction 1

2 The axion-like particle portal $\quad 3$

2.1 ALP effective Lagrangian 3

2.2 Astrophysical and cosmological constraints 5

2.3 ALPs production 8

3 Experimental searches for invisible ALPs 13

3.1 Limits from $e^{+} e^{-}$experiments 13

$\begin{array}{lll}3.2 & \text { Limits from electron beam dump experiments } & 18\end{array}$

3.3 Simulation of ALP production in the PADME environment 21

3.4 Magnetic moment of light leptons 25

4 Results $\quad 28$

4.1 Photo-philic or electro-philic ALP 28

$\begin{array}{lll}4.2 & \text { Combined case } & 29\end{array}$

5 Conclusions 33

\section{Introduction}

The Standard Model of particle physics (SM) has proven to be an extremely accurate description of nature, predicting with high precision practically all of the observables measured experimentally during the last decades. Yet, its shortcomings are equally well-known, for instance the nature of dark matter, the strong CP problem, the neutrino masses or the origin of the SM flavour structure. Over the years, a large number of Beyond Standard Model (BSM) solutions to these issues have been introduced, but so far none has been experimentally validated. While many of these models rely on introducing new particles around the $\mathrm{TeV}$ scale, this is neither a requirement nor, in many cases, the simplest possibility. New, light but feebly interacting particles, singlets under the SM gauge group, can often provide the most straightforward solution.

One of the best motivated possibilities for such new light particles are pseudo-NambuGoldstone Bosons (pNGB) a, pseudoscalar particles coupled very feebly to ordinary matter and radiation which arise from an explicit breaking of an approximate global symmetry in the UV. Lagrangians involving these fields enjoy an approximate shift symmetry under which $a \rightarrow a+$ const., which implies that their interactions has to proceed via derivative terms. According to the nature of the quasi-exact global symmetry, the corresponding pNGB is often denoted by a specific name: familon [1], majoron [2, 3], or axions [4-7]. More 
generally, light particles presenting the same types of derivative interactions are also motivated by various BSM theoretical scenarios, such as string theory [8, 9], or by extensions to the SM designed to address specific SM issues such as the strong-CP problem $[10,11]$ or the hierarchy problem [12].

From a bottom-up perspective, particles that enjoy the approximate shift symmetry mentioned above are often referred to as axion-like particles (ALPs). ${ }^{1}$ While the traditional mass range for such new particle typically extends in the extra-light, sub-eV region, the possibility of much more massive ALPs, in the MeV-GeV range, has received in recent years increasing attention (see. e.g. [13-23] for recent works on ALPs coupled to photons/leptons). ALPs in this mass range have been invoked for example as a possible solution for both the muon and the electron magnetic moment anomalies [24-30] and, interestingly, viable solutions to these anomalies require both electromagnetic and leptonic ALP interactions. Last but nor least, recent hints of the production of a $17 \mathrm{MeV}$ boson in nuclear transitions [31-34] have also been interpreted in terms of an hypothetical new pseudo-scalar particle $[23,35,36]$.

In this work we explore the possibility that an ALP acts as a portal towards a dark sector containing other invisible and light SM gauge singlets. Searches for such light and feebly interacting new particles can be carried out in experiments which trade a large energy scale for an increase in statistics and reduction in backgrounds [37, 38]. These so-called "high-intensity frontier" experiments have attracted a strong interest in recent years. Indeed, it has been know for a long time [39] that for interactions mediated by non-renormalisable operators of dimension $4+n(n=1, \ldots)$, when the energy scales of the dark sector physics is accessible, fixed targets experiments may very well surpass colliders in sensitivity. Among various well established search strategies, a particularly powerful one is the search for missing energy in fixed target and beam-dump experiments, as it does not require any particular assumption on the underlying structure of the dark sector.

In this work we focus on updating the current limits derived from missing energy searches for an ALP-portal scenario, assuming that the ALP decays dominantly invisibly so that most of the existing limits from visible decays searches do not apply. Furthermore, in simplified ALP models generally used to put experimental limits, it is often assumed that ALPs have only one type of interaction, for instance a coupling to the photons. Here we work in the more general scenario of a MeV-scale ALP coupled to both photons and electrons. Our aim is to capture the general phenomenological aspects of an ALP with independent couplings to electron and photons, studying correlations between processes induced by the different interactions, with particular attention to possible changes in the existing limits. We cover all available missing-energy searches, including high-energy results from the DELPHI [40] as well as high-intensity frontier experiments as BaBar [41] and NA64 [42], and we also present projections for Belle-II [43]. As an illustration of the effect of adding an invisible decay channel for standard long-lived ALP beam dump searches, we further study the limit from the E137 experiment [44] as function of the ALP invisible

\footnotetext{
${ }^{1}$ The name 'ALP' is inspired by the QCD axion, the difference being that ALPs are not required to solve the strong CP problem, in which case the mass and couplings of the ALP to fermions and photons can be freely taken as independent parameters.
} 
branching ratio. We show that a large suppression of the visible decay rate is required to alleviate the constraints from this class of experiments. Finally, we discuss the prospects for exploring ALP models of this type at the Positron Annihilation into Dark Matter Experiment (PADME) [45, 46] located in the Laboratori Nazionali di Frascati (LNF) in Italy. As PADME is based on a fixed-target positron beam accelerator setup, its search techniques differ significantly from other missing mass experiments. Near future upgrades of this experiment (and of the serving beam infrastructure [47]) will be able to probe a parameter space region complementary to NA64. Our results also provide for the first time a complete assessment of the status of ALP based solutions to the $(g-2)_{e}$ and $(g-2)_{\mu}$ anomalies. Strikingly, we have found that ALPs with masses as low as a hundred MeV can still provide a viable solution, which lies within range of upcoming Belle-II results.

A minimal model realising the scenario of an ALP coupled to both photons and electrons will be presented in section 2. Details about the astrophysical limits and ALP production channels at accelerators will be also presented in this section. Section 3 is dedicated to a recast of existing limits from accelerator experiments, to present the details of our estimates for the electron and muon magnetic moments, and to a description of the possibilities for ALP searches at PADME. In section 4 we present a comparison between different limits, and finally in section 5 we draw our conclusions.

\section{The axion-like particle portal}

\subsection{ALP effective Lagrangian}

ALPs are naturally produced by the spontaneous breaking of a global symmetry at some large new physics scale $\Lambda$. We follow the effective field theory (EFT) approach and focus on the ALP interaction with leptons and photons after electroweak symmetry breaking. The effective Lagrangian thus contains

$$
\mathcal{L} \subset \frac{1}{2}\left(\partial_{\mu} a\right)\left(\partial^{\mu} a\right)-\frac{1}{2} m_{a}^{2} a^{2}+\frac{1}{4} g_{a \gamma} a F_{\mu \nu} \tilde{F}^{\mu \nu}+\sum_{l=e, \mu, \tau} \frac{g_{a l}}{2}\left(\partial_{\mu} a\right) \bar{l} \gamma^{\mu} \gamma^{5} l
$$

where $\tilde{F}^{\mu \nu}=1 / 2 \epsilon^{\mu \nu \alpha \beta} F_{\alpha \beta}$ with $\epsilon^{0123}=-1$. In the spirit of ALP phenomenology, we take the mass $m_{a}$ and the couplings $g_{a \gamma}$ and $g_{a l}$ to be independent parameters. The ALP interactions are described by dimension- 5 operators and carry dimension of inverse mass $\left(\mathrm{GeV}^{-1}\right)$. The last term in eq. (2.1) is often rewritten as $-i g_{a l} m_{l} a \bar{l} \gamma_{5} l$ after integrating by part and applying the equations of motion. However, in passing from the derivative to the pseudoscalar coupling an anomalous contribution to the ALP interaction with photons must be added to the second term in eq. (2.1) [13]. In the rest of this work we will assume that the couplings to quarks and gluon are either absent, or negligible compared to $g_{a \gamma}$ and $g_{a e}$ (see for instance [20] for a recent summary of the limits on those couplings). ${ }^{2}$ As regards the couplings to the heavier $\tau$ and $\mu$ leptons, they would yield additional visible decays

\footnotetext{
${ }^{2}$ We also assume that the $N \gamma$ mixed coupling arising from the ALP interaction in an SU(2)-preserving basis is negligible, corresponding to coupling the ALP to $B$ and $W$ vector boson with the same strength (see, e.g. [14]).
} 
channels which in our analysis are not relevant. However, when we will briefly consider the prospects for the muon $g-2, g_{a \mu}$ will clearly play a relevant role. We assume the presence of particles of a dark sector in which the ALP predominantly decays, therefore, besides the interactions in eq. (2.1), the ALP couples derivatively to an axial-vector current involving dark sector states $\mathcal{J}_{5, D}^{\mu}$ :

$$
\mathcal{L} \supset \frac{g_{a \chi}}{2}\left(\partial_{\mu} a\right) \mathcal{J}_{5, D}^{\mu}
$$

For example, for a dark Dirac fermion $\chi$ one has $\mathcal{J}_{5, D}^{\mu}=\bar{\chi} \gamma^{\mu} \gamma^{5} \chi$.

The decay widths into two-photons or into an electron-positron pair are given by

$$
\begin{aligned}
\Gamma_{\gamma \gamma} & =\frac{g_{a \gamma}^{2} m_{a}^{3}}{64 \pi} \\
\Gamma_{e^{+} e^{-}} & =\frac{g_{a e}^{2}}{8 \pi} m_{e}^{2} m_{a} \sqrt{1-\frac{4 m_{e}^{2}}{m_{a}^{2}}},
\end{aligned}
$$

while the invisible decay width into a pair of dark Dirac fermions reads:

$$
\Gamma_{\mathrm{inv}}=\frac{g_{a \chi}^{2}}{8 \pi} m_{\chi}^{2} m_{a} \sqrt{1-\frac{4 m_{\chi}^{2}}{m_{a}^{2}}}
$$

Due to the $m_{a}^{3}$ scaling of the two-photon decay width, it is clear that one typically expects the photonic channel to dominate the visible decays at large ALP masses, except in the case of a significant hierarchy between the photon and the electron couplings. Let us define the visible decay rate of the ALP as $\Gamma_{\mathrm{vis}}=\Gamma_{\gamma \gamma}+\Gamma_{e^{+} e^{-}}$. The main assumption in this work is dominance of the invisible decays, that is

$$
\Gamma_{\text {inv }} \gg \Gamma_{\text {vis }}=\Gamma_{\gamma \gamma}+\Gamma_{e^{+} e^{-}} .
$$

These partial decay rates have important implications for both visible and invisible decays. Indeed if the size of the detector (for example, the distance between the target and the calorimeter at fixed target experiments) is smaller than the decay length, long-lived particles can mimic an invisible decay event. The typical ALP decay length $\ell_{a}$ is given approximately by:

$$
\ell_{a} \sim 0.1 \mathrm{~m} \times\left(\frac{100 \mathrm{MeV}}{m_{a}}\right) \times\left[\left(\frac{g_{a \chi} m_{\chi}}{0.7 \cdot 10^{-6}}\right)^{2}+\left(\frac{g_{a \gamma} m_{a}}{2 \cdot 10^{-6}}\right)^{2}+\left(\frac{g_{a e} m_{e}}{0.7 \cdot 10^{-6}}\right)^{2}\right]^{-1} .
$$

where we have assumed $m_{a} \gg m_{\chi}, m_{e}$ and Dirac dark fermions as an example of a possible dark sector. From the above equation it is clear that invisible decays will dominate as long as:

$$
g_{a \chi} m_{\chi} \gg g_{a \gamma} m_{a}, g_{a e} m_{e} .
$$

Furthermore, detection in beam dump experiments searching for visible ALP decay will be severely restricted as long as the invisible decay length is too short, which could typically occur in the region of parameter space where $g_{a \chi} m_{\chi} \gg 10^{-5}$. 
Let us note that in the case of a significant hierarchy between $g_{a \gamma}$ and $g_{a e}$, the subdominant coupling will receive important one-loop contributions generated by the leading one. Given that the experimental limit are typically much stronger for $g_{a \gamma}$, it is interesting to consider the electron one-loop contribution proportional to $g_{a e} \alpha_{\mathrm{em}}$. Using the derivative ALP-electron interaction from eq. (2.1) we have [13]:

$$
\delta g_{a \gamma}=\frac{\alpha_{\mathrm{em}} g_{a e}}{\pi} B_{1}\left(\frac{4 m_{e}^{2}}{m_{a}^{2}}\right),
$$

where the scalar loop function $B_{1}$ is given by:

$$
B_{1}(\tau)=1-\tau f(\tau)^{2}, \quad f(\tau) \equiv\left\{\begin{array}{l}
\arcsin (1 / \sqrt{\tau}) \quad, \tau \geq 1 \\
{\left[\frac{i}{2} \log \frac{1+\sqrt{1-\tau}}{1-\sqrt{1-\tau}}+\frac{\pi}{2}\right] \quad, \tau<1,}
\end{array}\right.
$$

with $B_{1}(\tau) \rightarrow 1$ for $\tau \rightarrow 0$ and $B_{1}(\tau) \sim 1 /(3 \tau)$ for $\tau \gg 1$. For $m_{a} \gg m_{e}$ one obtains $\delta g_{a \gamma} \sim 0.001 g_{a e}$, and one can thus expect the decay into photons to dominate over the decays into electrons as long as $m_{a} \gtrsim 1 \mathrm{GeV}$. Note that, if the correction to the ALPphoton coupling is computed using instead the non-derivative coupling to the pseudoscalar electron density, one would find a result proportional to $B_{1}(\tau)-1$ which in the limit of large fermion mass $\tau \gg 1$ does not decouple. However, as was pointed out in [13], the surviving -1 precisely cancels the anomalous contribution that, as was mentioned below eq. (2.1), had to be added to the ALP-photon interaction term when passing from the derivative of the axial-vector current to the pseudoscalar electron density. In the rest of this work, we will assume the coupling $g_{a \gamma}$ to be a completely free parameter, keeping in mind that a ratio $g_{a \gamma} / g_{a e}$ smaller than $\sim 0.001$ is not completely natural, and requires a certain level of tuning of the relevant parameters.

\subsection{Astrophysical and cosmological constraints}

Relic density and CMB. An ALP decay channel into invisible particles of a dark sector, and the corresponding suppression of the branching ratios for visible decays, can relax the limits from laboratory experiments, as well as from the cosmological imprint in the Cosmic Microwave Background (CMB) that could arise from late time decays (see e.g. the recent study in ref. [48]). However, the presence of new, light, and feebly coupled degrees of freedom has other preeminent consequences in cosmology and especially in astrophysics.

If stable, dark sector particles may constitute all or part of the Dark Matter (DM) content of the universe, a possibility has been studied in the last decade by various groups. However, it is relatively hard to suppress the relic density to an acceptable level in vanilla freeze-out scenarios that exploit a higher-dimensional portal (early studies include refs. $[49,50])$. Moreover, an additional difficulty is that of evading CMB limits on latetime annihilating DM [51]. Indeed, a DM candidate with mass below $\sim 10 \mathrm{GeV}$ would still keep annihilating at the time of matter-radiation decoupling, and would produce sizeable distortions of the CMB spectral shape [52]. ${ }^{3}$

\footnotetext{
${ }^{3}$ Indirect detection limits, in particular from Fermi-LAT [53], also constrain the mass region above $\sim 200 \mathrm{MeV}$.
} 
Nevertheless, a number of ways out from these difficulties have been put forth throughout the years. One possibility is to add a secluded annihilation channel via a renormalizable operator to fix the correct relic density, and then select a type of final states that will decay sufficiently fast in order to avoid all Big Bang nucleosynthesis (BBN) and CMB constraints [54-57]). Another possibility, that was sketched for example in ref. [58], is to use the entropy dilution from the decay of heavier relics to suppress the large relic density of $\mathrm{keV}$-scale DM left over by inefficient annihilation via the higher-dimension portal. In this case a complete cosmological model should also include the dependency on the reheating temperature for the production of the heavy relics, which is also subject to specific constraints. More exotic cosmological scenarios, such as a late time phase transition, can additionally have strong effects on the resulting relic density (see e.g. [59, 60] and related literature). With a set of $a d$ hoc assumptions the minimal scenario outlined below eq. (2.2) with the dimension five ALP portal and only one dark fermion $\chi$ can also be rendered viable. For instance, ref. [14] assumed a resonance setup where $2 m_{\chi} \lesssim m_{a}$ boosts the annihilation rate at earlier times, while later on at the BBN and CMB epochs the resonant annihilation channel is quenched because of the lower temperatures. In such case the proper relic density can be obtained for $g_{a \gamma}$ couplings as low as $10^{-5} \mathrm{GeV}^{-1}$. For the lowest masses we will consider (a few $\mathrm{MeV}$ ), ALP and/or dark sector particle could remain in thermal equilibrium until the time of neutrinos decoupling and $\mathrm{BBN}$, and this would yield additional constraints, see e.g. [61-63]. In this work, given that we do not specify the detailed structure of the dark sector, we will simply assume that a mechanism exists that allows to escape possible constraints from DM overabundance or from other cosmological arguments.

SN1987 limits. The duration of the neutrino burst from the supernova SN1987A provides to this date one of the strongest limit on several types of light new particles. In fact, particles with mass up to several tens of $\mathrm{MeV}$ could be efficiently produced in the collapsed core of a SN, and if they can freely escape, they would increase the cooling rate of the SN core and thus shorten the duration of the neutrino burst. However, if the interactions of these new particles with the surrounding medium are not sufficiently feeble, they would remain trapped in the core of the proto-neutron star and would not contribute to the cooling [64, 65]. According to the recent study in ref. [66] this would for example happen for ALP-photon couplings $g_{a \gamma} \gtrsim 10^{-6}-10^{-5}$ while, to the best of our knowledge, no analogous limit has been derived for the case of a purely electrophilic ALP (this might be due to the fact that ALP-electron couplings are usually considered in combination with ALP-quark interactions, which are expected to dominate because of the induced couplings of the ALP to the nucleons). The above result for $g_{a \gamma}$ implies that for practically the whole parameter space relevant for this work, the ALP will remain trapped and thus SN1987A does not provide useful constraints. Only in the lowest part of parameter space $\left(g_{a \gamma} \lesssim 10^{-5} \mathrm{GeV}^{-1}\right)$ trapping via ALP-photon interaction might be avoided. However, due to the intrinsic difficulty in pinning down the precise boundary between the trapping and free streaming regimes, it is safer to assume that also this region remains at least marginally compatible with the SN bound. The limits on the ALP couplings to SM particles, however, are 
of little importance in our case, since the decay/inverse-decay process $a \leftrightarrow \chi \bar{\chi}$ changes dramatically the discussion. In fact, as was noted in [14], even when the ALP is trapped because of sizeable $g_{a \gamma}$ or $g_{a e}$ couplings, the dark fermions $\chi$ might still escape and drain energy from the SN core, because $\chi$-trapping is much more difficult to achieve due to the dimension-5 nature of the ALP portal. However, ref. [14] only considered the scattering processes $\bar{\chi} \chi \leftrightarrow \mathrm{SMSM}$ for which the rates are heavily suppressed. We find instead that in the regime in which the ALP is trapped, $\chi$ interactions with on-shell ALPs give the dominant contribution to reduce the $\chi$ mean-free-path and to keep them trapped.

The SN limits for light dark matter are typically derived in two-steps. First, one estimates the luminosity $\mathcal{L}_{\chi}$ of the black body emission of fermions from a $\chi$-sphere of radius $R_{\chi}$ defined, in analogy to the usual neutrino-sphere, as the boundary surface between the trapping and free streaming regimes. For a Dirac fermion $\chi$ we have (see e.g. [67]):

$$
\mathcal{L}_{\chi}=\frac{g_{\chi}}{2 \pi} \int_{m_{\chi}}^{\infty} d E \frac{E\left(E^{2}-m^{2}\right)}{e^{E / T\left(R_{\chi}\right)}+1} \stackrel{m_{\chi} \ll T}{\longrightarrow} \frac{7 g \pi^{3}}{240} R_{\chi}^{2} T\left(R_{\chi}\right)^{4},
$$

where $T$ is the temperature and $g$ the number of degrees of freedom of the emitted particle ( $g=4$ for a Dirac fermion). Using the core profile from [68] and the neutrino luminosity $\mathcal{L}_{\nu} \sim 3 \cdot 10^{52} \mathrm{erg} \mathrm{s}^{-1}[65,68]$ we obtain that the typical radius for which $\mathcal{L}_{\chi} \lesssim \mathcal{L}_{\nu}$ is $R_{\chi} \sim 20 \mathrm{~km}$ for $m_{\chi}$ around $10 \mathrm{MeV}$. The second step is that of estimating $R_{\chi}$ which, as anticipated, is mainly determined by the inverse-decay process $\chi \bar{\chi} \rightarrow a$, as function of $g_{a \chi}$. Using the narrow width approximation $\left(\Gamma_{a} \ll m_{a}\right)$ which is always very accurate for the weakly interacting $\chi$, the relevant cross-section reads:

$$
\sigma_{\mathrm{res}}=\frac{\pi g_{a \chi}^{2} m_{\chi}^{2}}{2 \sqrt{1-4 m_{\chi}^{2} / m_{a}^{2}}} \delta\left(s-m_{a}^{2}\right) \equiv \tilde{\sigma}_{\mathrm{res}} \delta\left(s-m_{a}^{2}\right) .
$$

The number of interactions a $\chi$ particle suffers along an outward trajectory from $R_{\chi}$ is:

$$
N_{\mathrm{int}}=\int_{R_{\chi}}^{R_{\mathrm{far}}} d r\left[\frac{g_{\chi}}{n_{\chi}\left(T_{R_{\chi}}\right)} \int d^{3} \Pi_{\chi} d^{3} \Pi_{\bar{\chi}} f_{\chi}\left(T_{R_{\chi}}\right) f_{\bar{\chi}}(T) 4 E_{\chi} E_{\bar{\chi}} \sigma_{\mathrm{res}}\right],
$$

where $d^{3} \Pi$ is the standard Lorentz-invariant phase-space, $R_{\chi}$ is the radius of the dark sector sphere determined above, $f_{\chi}=\left(\exp \left(m_{\chi} / T\right)+1\right)^{-1}$ is the Fermi-Dirac statistical distribution, and $R_{\mathrm{far}} \sim 100 \mathrm{~km}$ corresponds to the outer layers of the proto-neutron star, out of which the profiles are not accurate. Altogether, eq. (2.13) is simply the thermal average (taken at $T_{R_{\chi}}$ ) of the number of interactions that a fermion $\chi$ emitted from $R_{\chi}$ has with the surrounding halo of $\bar{\chi}$ 's to resonantly produce an ALP. Given the simple form of $\sigma_{\text {res }}$ and the symmetric structure in $\bar{\chi}$ and $\chi$, the integral can be evaluated straightforwardly. Using standard integration techniques from the dark matter playbook (see e.g. the nice appendices in [69]) we have

$$
d^{3} \Pi_{\chi} d^{3} \Pi_{\bar{\chi}}=\frac{\left|p_{\chi}\right|\left|p_{\bar{\chi}}\right|}{32 \pi^{4}} d E_{\chi} d E_{\bar{\chi}} d \cos \theta
$$

where $\theta$ is the angle between $\chi$ and $\bar{\chi}$, and $\left|p_{\chi}\right|,\left|p_{\bar{\chi}}\right|$ their three-momenta. Using also

$$
s=2 m_{\chi}+2\left(E_{\chi} E_{\bar{\chi}}-\left|p_{\chi}\right|\left|p_{\bar{\chi}}\right| \cos \theta\right)
$$


and absorbing the angular integration by means of the delta function in eq. (2.12), we obtain the simple expression:

$$
N_{\mathrm{int}}=\int_{R_{\chi}}^{R_{\mathrm{far}}} d r\left[\frac{g_{\chi}}{n_{\chi}} \int_{m_{\chi}}^{\infty} \int_{m_{\chi}}^{\infty} d E_{\chi} d E_{\bar{\chi}}\left(\frac{E_{\chi} E_{\bar{\chi}}}{\left(e^{E / T(r)}+1\right)\left(e^{E / T\left(R_{\chi}\right)}+1\right)} \Theta\left(E_{1}, E_{2}\right)\right) \frac{\tilde{\sigma}_{\mathrm{res}}}{16 \pi^{4}}\right],
$$

where $\Theta\left(E_{\chi}, E_{\bar{\chi}}\right)=1$ in the resonance region defined by $s=m_{a}^{2}$, and 0 outside. Requiring at least one inverse-annihilation $N_{\text {int }} \geq 1$, and taking as an example $m_{\chi}=m_{a} / 3$, leads to the trapping limit on the $\chi$-ALP coupling

$$
g_{a \chi} m_{\chi} \gtrsim 10^{-6}
$$

that holds for $m_{\chi}$ between $\sim 5-50 \mathrm{MeV} .{ }^{4}$ Although eq. (2.17) is just an order of magnitude estimate, that moreover has been derived for the specific case of a DM Dirac fermion, it clearly shows that trapping can be achieve with reasonable dark sector couplings (typically of the order of the experimental limit on $g_{a e}$, which is consistent with our initial assumption of ALP decays dominated by invisible channels). We can then conclude that in our case the ALP limits from SN1987A cannot be applied.

Altogether, we conclude that the limits from SN1987 cooling can be escaped by trapping the light dark matter in the proto-neutron star via its resonant annihilation into ALP. In the following we will thus simply assume that the dark coupling $g_{a \chi}$ is large enough to trap $\chi$ and will focus instead on the collider signatures directly. Note that since the dark matter still does travel before annihilating, one should more rigorously consider the energy flux mediated by this process, using quantities like the Rosseland mean opacity [65]. In that case, the precise structure of the dark sector should be fleshed out, in particular if it contains more than one field. Nonetheless, eq. (2.17) is several orders of magnitude below what will be typically required to maintain an invisible ALP while having $g_{a e}, g_{a \gamma}$ around the state of the art limits from laboratory based experiments, so we still expect the later constraint to the dominate.

As a final comment, recent works $[66,70]$ have further pointed to the possible importance of the dark sector particles in the description of the actual supernova explosion. Deriving actual constraints from such considerations would however require an actual simulation of the supernova including the presence of a dark sector.

\subsection{ALPs production}

ALPs can be produced through many processes in lepton-based experiments. Since we focus mostly on missing energy searches, we will typically require an additional photon final state to trigger on the events. The main production channels in lepton beam accelerators are shown in figure 1. For positron beam experiments or $e^{+} e^{-}$colliders, the dominant processes

\footnotetext{
${ }^{4}$ An alternative approach is that of estimating directly the mean free path for a particle $\chi$ of energy $E_{\chi} \gtrsim m_{a} / 2$ and of mass in the tens of $\mathrm{MeV}$ range, emitted from the $\chi$-sphere $R_{\chi}$, and to require a mean free path of the order of $1 \mathrm{~km}$. Following this procedure we have found a value of $g_{a \chi} m_{\chi}$ of the same order of magnitude than in eq. (2.17).
} 
are annihilation in the $t$ or $s$-channel $e^{+}+e^{-} \rightarrow \gamma+a$ (figure 1a and 1b), the former depending on the electron coupling $g_{a e}$ and the latter on the ALP-photon coupling $g_{a \gamma}$. In electron-beam experiments, the dominant processes are either ALP direct bremsstrahlung production on a target nucleus $N$ via the $g_{a e}$ coupling shown in figure 1c, or conversion of bremsstrahlung photons from $e^{-} N \rightarrow e^{-} N \gamma$ into ALPs via the process $\gamma N \rightarrow N a$ shown in figure $1 \mathrm{~d}$ that depends on $g_{a \gamma}$. Several other ALP production channels have been considered in the literature. However, they are either subdominant in the experimental setups we are considering here, or not relevant because they do not provide the final states that have been searched for by the experimental collaborations. For instance, the 'photonfusion' process $e^{+} e^{-} \rightarrow e^{+} e^{-}\left(\gamma^{*} \gamma^{*}\right) \rightarrow e^{+} e^{-} a$, which can have significant rates in $e^{+} e^{-}$ colliders $[13,25]$ does not lead to a mono-photon final state (unless a $\gamma$ is radiated from a fermion line, in which case the process is of higher order), and is subdominant with respect to Primakoff production in experiments such as NA64 in which the beam impinges onto a high- $Z$ dump. ALPs can be also produced via the inverse-Compton process $\gamma e^{-} \rightarrow a e^{-}$ that depends on $g_{a e}$. This could be an effective production channel in beam dumps where a large population of secondary photon is generally created [71, 72]. However, we have checked that inverse-Compton remains a sub-dominant production process with respect to ALP bremsstrahlung from the primary electrons of the beam. ${ }^{5}$

Positron-electron annihilation. We first focus on the associated annihilation processes, which is relevant for positron beam and $e^{+} e^{-}$experiments. The total annihilation cross-section in the laboratory frame is given by:

$$
\begin{aligned}
\sigma=\sigma_{a \gamma}+\sigma_{a e}+\sigma_{\mathrm{int}}= & \alpha_{\mathrm{em}} g_{a \gamma}^{2} \frac{\left(s+2 m_{e}^{2}\right)\left(s-m_{a}^{2}\right)^{3}}{24 \beta s^{4}} \\
& +\alpha_{\mathrm{em}} g_{a e}^{2} m_{e}^{2} \frac{-2 m_{a}^{2} \beta s+\left(s^{2}+m_{a}^{4}-4 m_{a}^{2} m_{e}^{2}\right) \log \frac{1+\beta}{1-\beta}}{2\left(s-m_{a}^{2}\right) s^{2} \beta^{2}} \\
& +\alpha_{\mathrm{em}} g_{a \gamma} g_{a e} m_{e}^{2} \frac{\left(s-m_{a}^{2}\right)^{2}}{2 \beta^{2} s^{3}} \log \frac{1+\beta}{1-\beta}
\end{aligned}
$$

where $\alpha_{\mathrm{em}}=e^{2} /(4 \pi)$ is the electromagnetic fine structure constant, $m_{e}$ the electron mass, $\beta=\sqrt{1-\frac{4 m_{e}^{2}}{s}}$ and $\sqrt{s}$ is the center-of-mass energy. In the first equality $\sigma_{a \gamma}, \sigma_{a e}$ and $\sigma_{\text {int }}$ refer respectively to the contributions to the annihilation from $s$-channel photon exchange (figure $1 \mathrm{~b}$ ), $t$ - and $u$-channel electron exchange (figure 1a), and to their interference. The plots in figure 2 show the different behaviours of $\sigma_{a \gamma}$ and $\sigma_{a e}$ as a function of the ALP mass $m_{a}$ for various beam energies. Interestingly, the two processes have a starkly different

\footnotetext{
${ }^{5} \mathrm{~A}$ special case is positron annihilation on atomic electrons via the diagram in figure 1a when $2 m_{e} E_{e^{+}} \approx m_{a}^{2}$ and the emitted photon is soft. However, this process can be seen as a radiative correction to the resonant annihilation $e^{+} e^{-} \rightarrow a$ which then should be also included. The importance of resonant annihilation for positron beam fixed target experiments was first pointed out in ref. [73] in relation to dark photon searches at PADME, and was later included in a reanalysis of electron beam experiments in which the positrons arise as secondaries [74, 75]. At NA64, given the strong energy cut of $50 \mathrm{GeV}$, resonant annihilation would typically contribute for $m_{a} \sim 200-300 \mathrm{MeV}$, and could possibly help the experiment to overcome BaBar constraints in this narrow range. A dedicated study of this possibility, similarly to what has been done for instance for E137 [75], could indeed be interesting, and is left for future work.
} 


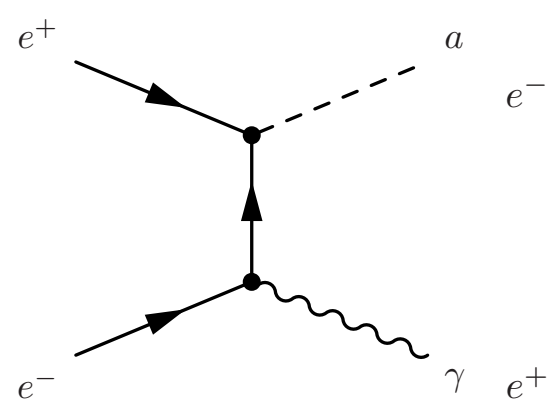

(a)

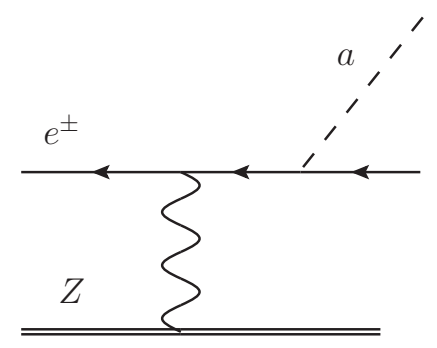

(c)

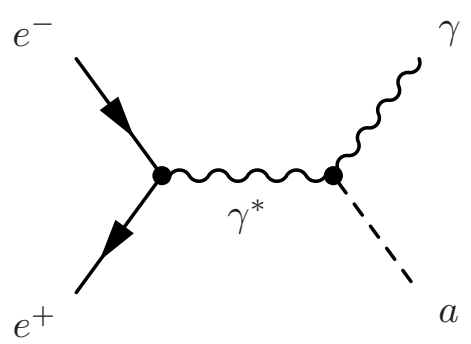

(b)

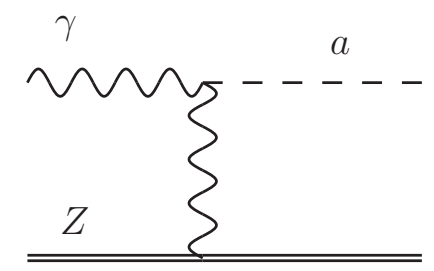

(d)

Figure 1. Feynman diagrams for ALP production: (a) $t$-channel annihilation process $e^{+} e^{-} \rightarrow \gamma+a$; (b) $s$-channel annihilation process $e^{+} e^{-} \rightarrow \gamma+a$; (c) Bremsstrahlung production off a target nucleus $N$ of atomic number $Z, e^{ \pm} \rightarrow N+e^{ \pm}+a$; (d) Primakoff production from a secondary photon $\gamma+N \rightarrow N+a$.



Figure 2. Contributions to the $e^{+} e^{-}$annihilation cross-section as a function of the ALP mass $m_{a}$ for three different values of the beam energy $E_{\text {beam }}=0.55,1,10 \mathrm{GeV}$. Continuous lines refer to $t$ - and $u$-channel electron exchange (figure 1a) with $g_{a e}=1 \mathrm{GeV}^{-1}$. Dotted lines to $s$-channel photon exchange (figure 1b) with $g_{a \gamma}=1 \mathrm{GeV}^{-1}$. 
behaviour, due to the presence of a resonant enhancement in the soft-photon limit in the $t$ and $u$-channel processes of figure 1a. On the other hand, the electron and photon exchange contributions are roughly of the same order when the two couplings $g_{a \gamma}$ and $g_{a e}$ satisfy

$$
g_{a \gamma} \approx g_{a e} \frac{m_{e}}{\sqrt{s}} .
$$

This relation underlies much of the phenomenology of the next sections: photon-mediated ALP production is relevant at large center-of-mass energies, while the converse is true for electron-mediated processes. In particular, the associated ALP- $\gamma$ production is characterised by a resonant enhancements when $\sqrt{s} \sim m_{a}$ [73]. This implies that high-statistics low-energy positron-beam experiments can be particularly efficient in constraining the ALP-electron coupling since they can exploit the resonant process to enhance dramatically the production of ALPs. As regards the interference term in eq. (2.18), it presents the downsides of both the pure electron and pure photon contributions, and is subdominant in all the parameter space we have probed. Indeed, considering the "maximum interference" scenario in which $g_{a \gamma}$ and $g_{a e}$ fulfill the condition in eq. (2.19) we see that $\sigma_{\text {int }}$ does not get resonantly enhanced at $\sqrt{s} \sim m_{a}$, and that in the large $\sqrt{s} \gg m_{a}$ limit it scales with an additional suppression factor of $m_{e} / \sqrt{s}$.

Bremsstrahlung production. Let us now consider the most relevant production channels for electron beam experiments. In this case, ALP production relies on the electron or photon interaction with the electromagnetic field sourced by an atomic nucleus $N$. In the first case the electron interacts with the nucleus via a virtual photon, and recoils emitting an ALP via bremsstrahlung. The physics underlying this process has been known for several decades (see [76] for a review of early studies). The photon interaction with the nucleus is described in terms of a form factor with one elastic and one inelastic component: $G_{2}(t)=G_{2}^{\mathrm{el}}+G_{2}^{\mathrm{in}}$. Denoting with $t$ the virtuality (i.e. the squared momentum) of the virtual photon, we have: 6

$$
\begin{aligned}
G_{2}^{\mathrm{el}} & =\left(\frac{a^{2} t}{1+a^{2} t}\right)^{2}\left(\frac{1}{1+t / d}\right)^{2} Z^{2}, \\
G_{2}^{\mathrm{in}} & =\left(\frac{a^{\prime 2} t}{1+{a^{\prime}}^{2} t}\right)^{2}\left(\frac{1+\frac{t}{4 m_{p}^{2}}\left(\mu_{p}^{2}-1\right)}{\left(1+\frac{t}{0.71 \mathrm{GeV}^{2}}\right)^{4}}\right) Z,
\end{aligned}
$$

where $Z$ is the nucleus atomic number, $m_{p}=0.938 \mathrm{GeV}$ is the proton mass, $\mu_{p}=2.79$ is the nuclear magnetic dipole moment. The parameters $a[79]$ and $a^{\prime}[76]$ are related to the atomic size in the elastic and inelastic case, and are determined such that in the limit of complete screening one reproduces the numerical results, while $d$ parametrises the inverse nucleon square radius. Their values are $[76,79]$

$$
a=111 \frac{1}{m_{e} Z^{1 / 3}}, \quad a^{\prime}=773 \frac{1}{m_{e} Z^{2 / 3}}, \quad d=0.164 \mathrm{GeV}^{2} A^{-2 / 3} .
$$

\footnotetext{
${ }^{6}$ Note that our expression for $G_{2}^{\text {in }}$ differs from the one given in eq. (A19) of ref. [77] due to a spurious square for the term in the second parenthesis appearing in that reference, see also ref. [78].
} 
As noted in ref. [80], the exchange of a very soft or very hard photon is suppressed due to either the screening from the electrons in the atomic cloud when $a^{2} t, a^{\prime 2} t \ll 1$ or from the finite nuclear size in the opposite limit $t \gg d$.

Although both the $g_{a \gamma}$-driven Primakoff and $g_{a e}$-driven bremsstrahlung processes share the same effective interaction with the target nuclei, the former is strongly enhanced with respect to the latter. Indeed, in the regime $m_{e} \ll m_{a} \ll E_{0}$ (with $E_{0}$ the incoming electron energy) one has the scaling:

$$
\begin{aligned}
& \sigma_{a e} \propto \alpha_{\mathrm{em}}^{2} g_{a e}^{2} \frac{m_{e}^{2}}{m_{a}^{2}}, \\
& \sigma_{a \gamma} \propto \alpha_{\mathrm{em}} g_{a \gamma}^{2},
\end{aligned}
$$

so that the bremsstrahlung cross-section is suppressed with respect to Primakoff production by the ratio $m_{e}^{2} / m_{a}^{2}$, by an extra factor of $\alpha_{\mathrm{em}}$, and by an additional suppression factor from three-body vs. two-body phase space that is left implicit in eqs. (2.22), (2.23). It is also worth noting that, for equal values of the masses and up to order one factors from the different nature of the outgoing particles, under the exchange:

$$
e \varepsilon \leftrightarrow g_{a e} m_{e},
$$

the cross section for ALP production via bremsstrahlung is equivalent to that for bremsstrahlung production of dark photons. This reflects a generic feature that will prove to be quite useful in our work: the electron $t$-channel ALP production closely mimics the results for the dark photon.

The number of ALP produced in a given electron beam dump experiments is given by:

$$
\mathcal{N}=\frac{\mathcal{N}_{A} X_{0} \rho}{A} \int_{E_{c}}^{E_{0}} d E \frac{\partial T_{e / \gamma}}{\partial E} \int_{E_{c}}^{E} d E_{a} \frac{d \sigma}{d E_{a}} \equiv \frac{\mathcal{N}_{A} X_{0} \rho}{A} \sigma_{\mathrm{eff}}
$$

where we have accounted for the possibility of an experimental energy cut-off $E_{c}$ on the energy $E_{a}$ of the emitted ALP, and $\mathcal{N}_{A}=6 \times 10^{23} \mathrm{~mole}^{-1}$ is the Avogadro number, $A$ is the atomic number of the dump medium in $\mathrm{g} /$ mole, $X_{0}$ its radiation length in $\mathrm{cm}, \rho$ its mass density in $\mathrm{g} \mathrm{cm}^{-3}, T_{e / \gamma}$ the electron or photon track length, and the cross-section $\sigma_{\text {eff }}$ goes in units of $\mathrm{cm}^{2}$. In the last equality we have defined an "effective" cross-section, which includes the integration over $\partial T_{e / \gamma} / \partial E$. These differential track lengths represent the total length (in unit of radiation length) of material traversed by all $e^{ \pm}$or $\gamma$ of a given energy $E$ present in the shower. While the track lengths can be obtained directly via a Monte Carlo (MC) simulation of the shower, a relatively reliable expressions for electron beam dump has been derived long ago in [81]:

$$
\frac{\partial T_{e / \gamma}}{\partial E}=\int_{0}^{t_{\mathrm{tar}}} d t I_{e / \gamma}(t, E), \text { where }\left\{\begin{array}{l}
I_{e}(t, E)=\frac{1}{E} \frac{\left.\left[\ln \left(E_{0} / E\right)\right)\right]^{4 t / 3-1}}{\Gamma(4 t / 3)} \\
I_{\gamma}(t, E)=\frac{1}{E} \frac{\left(1-E / E_{0}\right)^{4 t / 3}-e^{-7 t / 9}}{\frac{7}{9}+\frac{4}{3} \ln \left(1-E / E_{0}\right)}
\end{array}\right.
$$


where $t_{\operatorname{tar}}$ is the target length in unit of radiation length. We have simulated both production processes in MadGraph5_aMC@NLO using an effective $N-\gamma$ interaction with form factor $G_{2}$. This implies that we did not use the Weizsacker-Williams approximation for the cross section [82], but we have instead estimated directly the $2 \rightarrow 3$ bremsstrahlung and $2 \rightarrow 2$ Primakoff processes. Furthermore, in order to regulate the numerical divergence which arises for large electron energies when the exchanged photon is very soft, we have modified the form factor $G_{2}(t)$ : we know that, due to the screening effects occurring when $a^{2} t \ll 1$, this part of the phase space is sub-dominant in the final production rate. We have therefore imposed a regularising cut by setting the form factor to 0 in the "screened" region:

$$
G_{2}^{r}(t)= \begin{cases}G_{2}(t) & \text { for } a^{2} t>1 / 3 \\ 0 & \text { for } a^{2} t<1 / 3 .\end{cases}
$$

We have explicitly checked that the value of the cross section is not modified by varying the cut between $a^{2} t<1$ and $a^{2} t<0.05$, and that it agrees with the analytical expression developed above. Furthermore, we have verified that the differential distribution in angles and energy are also not affected by this regularisation.

We conclude this section by illustrating in figure 3 the hierarchy between both production modes in the case of the NA64 beam dump (that will be thoroughly studied in the next section). As expected, the photon-driven production strongly dominates the total rate, even though photons are secondary particles in the $e^{-}$-initiated electromagnetic shower. This is due in part to the strong enhancement of the Primakoff production rate compared to bremsstrahlung, and in part from the fact that bremsstrahlung photons carry a large fraction of the energy of the scattered primary electrons and, being the NA64 target around 40 radiation lengths all primary electrons will eventually undergo such a process.

\section{Experimental searches for invisible ALPs}

A trademark of the scenario which is the subject of this work is that, in contrast with standard long-lived ALP searches, here the invisible channel is assumed to dominate the ALP decay in all regions of parameter space. The signatures to search for such an ALP can be divided between "pure" missing energy searches, such as the ones performed by NA64, where one directly triggers on very low energy events created from high-energetic primaries, and "associated" missing energy searches, where the experiments use a single photon produced in association with the ALP to search for bumps on its recoil mass.

\subsection{Limits from $e^{+} e^{-}$experiments}

A first class of experiments rely on $e^{+} e^{-}$colliders to produce the ALP via the process $e^{+} e^{-} \rightarrow a \gamma$, and trigger on single photon events with a large missing energy from the escaping ALP. We will consider the re-interpretation of searches carried out in the BaBar [41] and DELPHI [40] experiments, as well as projections for the Belle-II sensitivity [43]. Due the ALP decaying mainly invisibly, the LEP constraints [83] on the visible mode $e^{+} e^{-} \rightarrow \gamma \gamma$ from are not relevant here. 


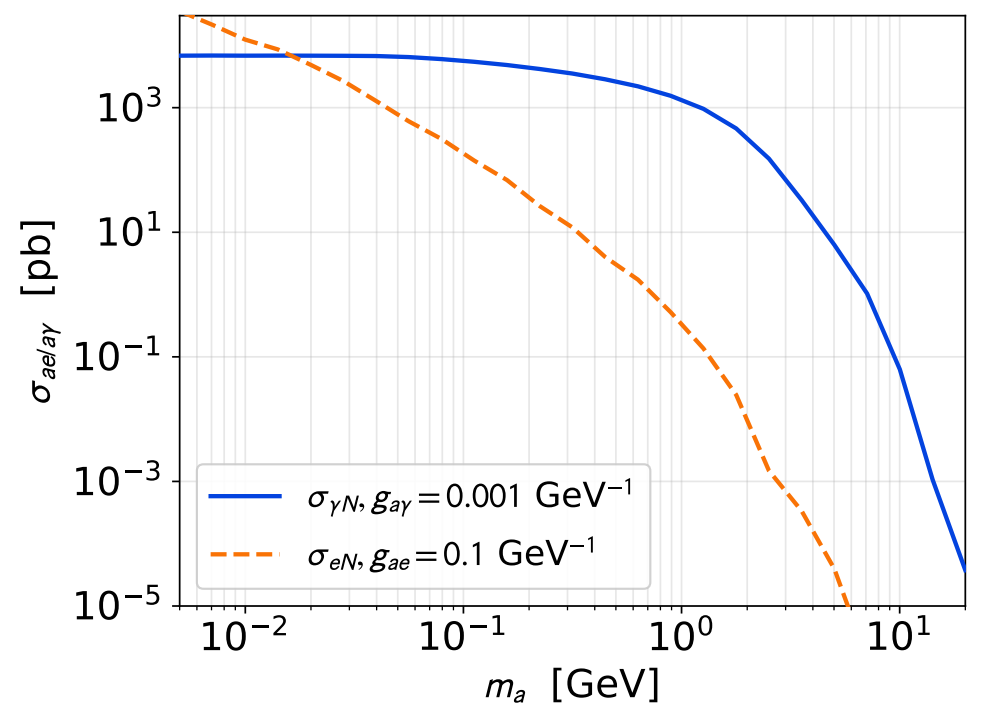

Figure 3. Effective production cross-section for the Primakoff (solid blue line) and bremsstrahlung (dashed red line) processes in NA64. The initial electron/photon energy is weighted by the corresponding track length distribution $\partial T_{e / \gamma} / \partial E$ in the electromagnetic shower.

As was discussed in the previous sections, the $g_{a e}$ and $g_{a \gamma}$ couplings provide two diagrams for ALP production that, due to the suppression of interference effects, to a very good approximation correspond to two independent production processes. We can then define the total production rate times the experimental efficiency $(\epsilon \sigma)_{e^{+} e^{-} \rightarrow a \gamma}$ as the sum of the corresponding partial cross sections:

$$
(\epsilon \sigma)_{e^{+} e^{-} \rightarrow a \gamma}=g_{a e}^{2}(\varepsilon \sigma)_{e}+g_{a \gamma}^{2}(\varepsilon \sigma)_{\gamma},
$$

where we have put in evidence the $g_{a \gamma}$ and $g_{a e}$ dependence of the two contributions. Since all the experiments that we will consider follow a "cut-and-count" search strategy, we can estimate the limits $g_{a e}^{\lim }$ and $g_{a \gamma}^{\lim }$ from the two production process independently, and then combine both rates to obtain coupled limits as

$$
\left(g_{a e}, g_{a \gamma}\right)^{\lim } \leqslant\left(\left(\frac{g_{a e}^{1}}{g_{a e}^{\lim }}\right)^{2}+\left(\frac{g_{a \gamma}^{1}}{g_{a \gamma}^{\lim }}\right)^{2}\right)^{-1 / 2} \times\left(g_{a e}^{1}, g_{a \gamma}^{1}\right),
$$

where the pair of couplings $\left(g_{a e}^{1}, g_{a \gamma}^{1}\right)$ is used as an input to fix a priori a specific ratio between the two independent couplings. We will use the above approach for the DELPHI and PADME limits, for which we have fully simulated the expected signal. For BaBar (and for the Belle-II projection), we will instead rely on comparing directly $(\epsilon \sigma)_{e^{+} e^{-} \rightarrow a \gamma}$ with the limits on dark photon searches reported (projected) by these two experiments.

LEP-DELPHI. The DELPHI experiment at LEP has performed a single-photon search [40] for the emission of an invisible graviton from low-scale extra-dimension. Starting with ref. [84], the results of this search have been widely recasted to constrain more 


\begin{tabular}{|c|cccccccccc|}
\hline$\langle\sqrt{s}\rangle(\mathrm{GeV})$ & 182.7 & 188.6 & 191.6 & 195.5 & 199.5 & 201.6 & 203.7 & 205.2 & 206.7 & 208.2 \\
\hline $\mathcal{L}\left[\mathrm{pb}^{-1}\right]$ & 50.2 & 154.7 & 25.9 & 76.4 & 83.4 & 40.6 & 8.4 & 76.2 & 121.6 & 8.3 \\
\hline
\end{tabular}

Table 1. Luminosity acquired in the DELPHI HPC detector as function of the average CoM energy delivered by LEP [86].

generic types of invisible particles. Using CalcHEP [85] we have simulated $10^{4} e^{+} e^{-} \rightarrow \gamma+a$ events, and we have followed [40] and [84] in imposing the following cuts

$$
45^{\circ}<\theta_{\gamma}<135^{\circ} \text { and } 0.9<E_{\gamma} / E_{\text {beam }}<1.05,
$$

where $\theta_{\gamma}\left(E_{\gamma}\right)$ is the photon angle (energy) in the center-of-mass frame. Note that there is no correlation between $E_{\gamma}$ and $\theta_{\gamma}$, as is clear analytically since $E_{\gamma}=\left(s-m_{a}^{2}\right) /\left(4 E_{\text {beam }}\right)$. The DELPHI experiment quotes an energy resolution [40]:

$$
\frac{\sigma_{E}}{E_{\gamma}}=0.0435 \oplus \frac{0.32}{\sqrt{E_{\gamma}}},
$$

where $\oplus$ denote the sum of two independent Gaussian distributions. This leads to a significant smearing of an otherwise narrow distribution centered around $E_{\gamma}=\left(s-m_{a}^{2}\right) /\left(4 E_{\text {beam }}\right)$, and is particularly important to model properly the threshold in $m_{a}$ induced by the energy cuts $0.9<E_{\gamma} / E_{\text {beam }}<1.05$. Altogether, these cuts lead to a selection efficiency of around $40 \%$ for, e.g. $m_{a}=10 \mathrm{MeV}$ and $g_{a \gamma}=0.1 \mathrm{GeV}^{-1}$.

The full DELPHI data sample was collected at different CoM energies, ranging from $180.8 \mathrm{GeV}$ to $209.2 \mathrm{GeV}$ [86]. The corresponding accumulated luminosity per energy bin is given in table 1. Although the change in CoM energy was not relevant for ref. [84], which considered off-shell new physics operators whose effects were spread in the various energy bins, in our case it is important to model properly the various mass thresholds. We have therefore estimated the efficiency for the energy cuts as a weighted sum over the integrated luminosity per energy bin. Finally, following [84] we have also included an overall trigger efficiency $\varepsilon_{t}=84 \%$. This yields the full efficiency:

$$
\varepsilon_{\mathrm{DELPHI}}=\varepsilon_{\theta} \times \varepsilon_{t} \times \sum_{i} \frac{\mathcal{L}_{i}}{\mathcal{L}_{\mathrm{tot}}} \varepsilon_{s_{i}}
$$

where $\varepsilon_{s_{i}}$ is the efficiency of the energy cuts for a LEP CoM energy $\sqrt{s_{i}}$ and luminosity $\mathcal{L}_{i}$ as given in table $1, \varepsilon_{\theta}$ is the efficiency of the angular cut, which depends on the ALP production process, and $\mathcal{L}_{\text {tot }}=645.7 \mathrm{pb}^{-1}$ is the total integrated luminosity. The efficiencies of the angular and energy cuts for the two main ALP production mechanisms are plotted in figure 4 . The dashed orange line represents the efficiency for the $t$-channel associated production assuming that the entire luminosity is collected at the average CoM energy $\langle\sqrt{s}\rangle=200 \mathrm{GeV}$, while the orange solid line corresponds to the more accurate result obtained by accounting for the CoM energy of each partial integrated luminosity. We see that while there is good agreement for small ALP masses, the results differ significantly for an ALPs mass exceeding $m_{a} \sim 70-80 \mathrm{GeV}$. The solid blue line in the figure depicts the 


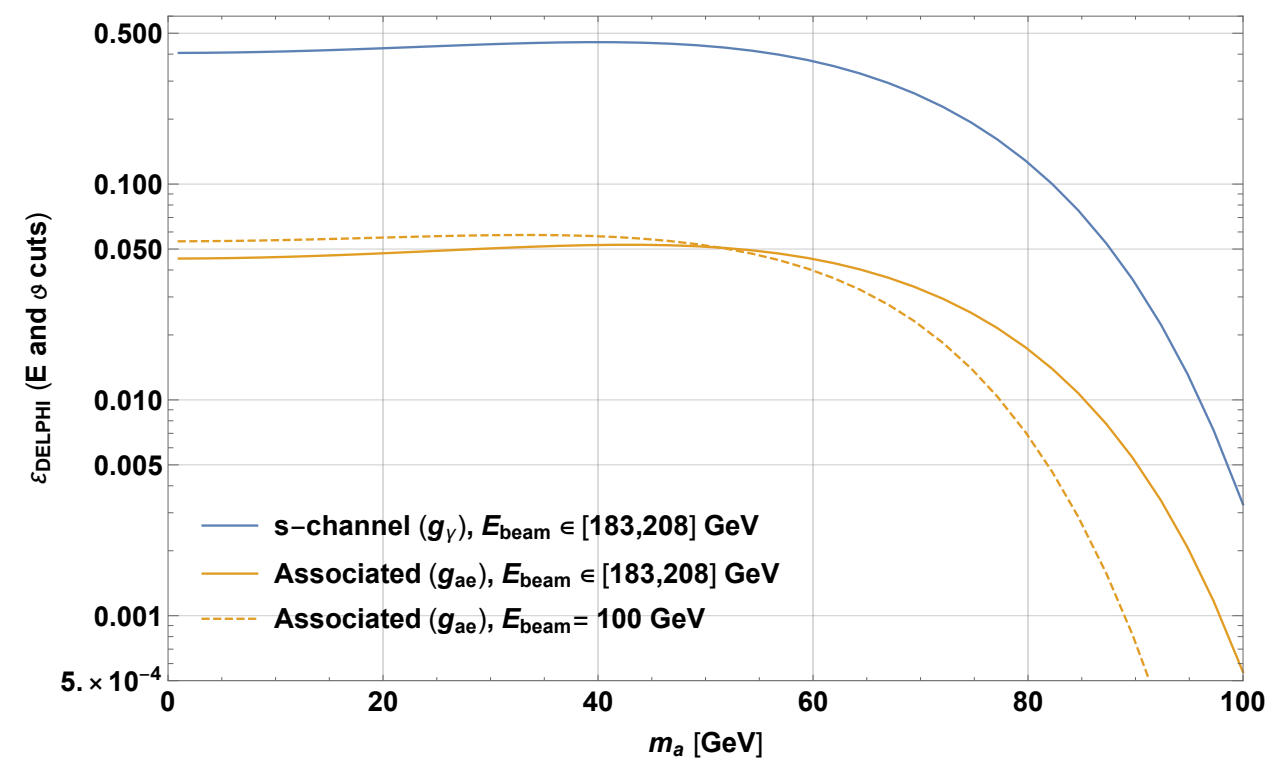

Figure 4. Estimated total efficiencies for the energy cut $0.9<E_{\gamma} / E_{\text {beam }}<1.05$ and angular cut $45^{\circ}<\theta_{\gamma}<135^{\circ}$ at DELPHI, for the $s$-channel ALP production process controlled by $g_{a \gamma}$ (orange continuous line) and for the associated production controlled by $g_{a e}$ (blue line). The dashed orange line correspond to the efficiency for the associated production in the case the CoM energy is fixed at the average value $\langle\sqrt{s}\rangle=200 \mathrm{GeV}$.

complete result for the photon $s$-channel process. It can be seen that the overall efficiency for this channel is much higher than that for the associated production. This is due to the angular cuts, and reflects the different shape of the angular differential distribution that, for the associated production, is peaked at small angles. For the statistical analysis of ALP production we need to compare the simulated signal events with the collected data. One additional complication is that, especially in the last bin, the agreement between the predicted number of background events from the MC simulation in [40] and the actual data is rather poor. However, the simulation of $e^{+} e^{-} \rightarrow \gamma \nu \bar{\nu}$ events performed a posteriori in [84] predicted around 49 such background events for $E_{\gamma} / E_{\text {beam }}$ in the interval $[0.9,1.05]$, in better agreement with the 61 events observed. Altogether, a first conservative approach is to assume that every observed event originates from an ALP, setting the limit on the number of observed events to $N_{\lim }^{90} \sim 72$ at $90 \%$ confidence level (C.L.). A more aggressive approach is instead to account for the $e^{+} e^{-} \rightarrow \gamma \nu \bar{\nu}$ simulation from [84], use an overall $10 \%$ uncertainty on this background (to be compared to the $5 \%$ systematic uncertainty quoted in [86]), and to assume that the remaining events are produced by ALPs. This leads instead to $N^{90} y_{\text {lim }} \sim 25.5$ at $90 \%$ C.L., and to a $\sim 40 \%$ stronger limit with respect to the conservative approach. In the next sections, when providing the final limits, we will adopt the latter choice, keeping in mind that this stems from somehow aggressive assumptions. Once $g_{a e}^{\lim }$ and $g_{a \gamma}^{\lim }$ have been obtained for both ALP production processes as function of the ALP mass $m_{a}$, they can eventually be combined as in eq. (3.2).

BaBar. The BaBar experiment at the PEP-II B-factory has accumulated a large dataset of $e^{+} e^{-}$collision events, corresponding to a total luminosity of $53 \mathrm{fb}^{-1}$. Single photon 
events with large missing energy were analysed via a bump-search [41], focusing on the case of a dark photon. ${ }^{7}$

As such, the limits reported by the collaboration need to be modified to account for the different production channels and kinematics of the ALP case. We have use the CalcHEP simulation setup described above, tuned to the BaBar characteristics: an asymmetric interaction between $9 \mathrm{GeV}$ electrons and $3.1 \mathrm{GeV}$ positrons to produce a CoM collision energy of $10.58 \mathrm{GeV}$. The selection cuts on the CoM photon angle

$$
\begin{array}{ll}
-0.4<\cos \theta_{\gamma}<0.6 & \text { for } m_{a}<5.5 \mathrm{GeV} \\
-0.6<\cos \theta_{\gamma}<0.6 & \text { for } m_{a}>5.5 \mathrm{GeV},
\end{array}
$$

were applied directly to the MC truth events using Root [88]. Note that BaBar selection cuts further require $E_{\gamma}>3(1.5) \mathrm{GeV}$ for the low (high) mass search, but this cuts are redundant because of the angular ones up to a threshold mass of about $m_{a} \sim 8 \mathrm{GeV}$. We plot in figure 5 the energy and the emission angle distributions for a dark photon and for the two ALP production processes. As expected, the two cases have a priori very distinct differential distributions. Note that since the photon energy in the CoM frame is fixed as $E_{\gamma}^{C o M}=\left(s-m_{a}^{2}\right) /(2 \sqrt{s})$, the correlation between the energy and the angle in the laboratory frame is of purely kinematic origin, and does not depend on the nature of the emitted particle. On the other hand, as can be seen from figure 5 , the efficiencies of the geometric cuts differs strongly for the two cases and need to be fully accounted for. Note that the full BaBar analysis uses a Boosted-Decision-Tree (BDT) approach, whose efficiency as function of the signal characteristics $\left(E_{\gamma}\right.$ and $\left.\theta_{\gamma}\right)$ is not reported. However, as is clear from figure 5 , the actual distribution of signal events within the angular cuts differ (up to an overall normalisation factor) by at most $25 \%$. Since the BDT only considers the signal on an event-per-event basis, a BDT variation in efficiency between the two cases cannot be significantly larger than this number, which is then included as an additional uncertainty in our final result. In order to extend the limits from [41] to the case of an ALP with both $g_{a \gamma}$ and $g_{a e}$ couplings, we have calculated the cross-section times efficiencies for $e^{+} e^{-} \rightarrow \gamma a$ in the acceptance region of BaBar as function of the ALP mass. We then estimate the ratio of this quantity with the corresponding one for the production of a dark photon $V$ of the same mass $m_{V}=m_{a}$ and for a kinetic mixing $\varepsilon=10^{-3}$ :

$$
R\left(g_{a \gamma}, g_{a e}, m_{a}\right) \equiv \frac{(\epsilon \sigma)_{e}+(\epsilon \sigma)_{a}}{(\epsilon \sigma)_{e^{+} e^{-} \rightarrow \gamma V}} .
$$

The limit on the ALP couplings is then given by:

$$
\left(g_{a e}^{\lim }, g_{a \gamma}^{\lim }\right)=\left(\frac{\varepsilon_{\mathrm{BaBar}}}{0.001}\right) \frac{1}{\sqrt{R\left(m_{a}\right)}},
$$

where $\varepsilon_{\mathrm{BaBar}}$ is the limit reported by the collaboration. The final result only depends on the ratio between the ALP couplings $g_{a e} / g_{a \gamma}$ so that the ratio $R$ can be evaluated by keeping the sum of the square of the couplings fixed to some specific value $g_{a e}^{2}+g_{a \gamma}^{2}=\bar{g}^{2}$.

\footnotetext{
${ }^{7}$ The BaBar collaboration had previously carried out also a search for production of invisible final states in single-photon decays of $\Upsilon(1 S)$ [87], which however yields limits that are less stringent than those implied by their more recent search [41] on which we focus here.
} 

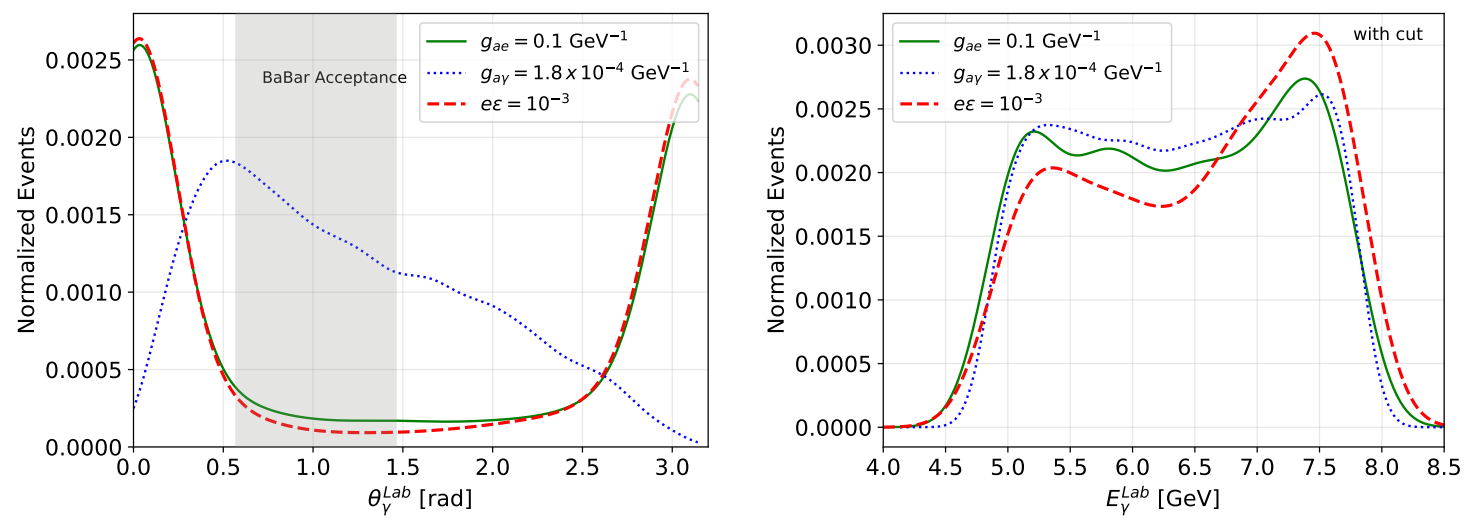

Figure 5. Normalized photon emission angle distribution (left panel) and energy distribution with BaBar angular cuts (right panel) in the laboratory frame for $e^{+} e^{-} \rightarrow \gamma X$ with $X=$ dark photon, ALP and $M_{X}=20 \mathrm{MeV}$. The red dashed line is for a dark photon with a coupling $e \epsilon=10^{-3}$, the green line for an ALP with $g_{a e}=10^{-3} \mathrm{GeV}^{-1}$, and the blue dotted line for an ALP with $g_{a \gamma}=1.8 \times 10^{-4} \mathrm{GeV}^{-1}$. The grey region represents the BaBar angular coverage.

Prospects for Belle-II. The experiment Belle-II [43] is currently accumulating data and in the coming years is expected to produce significantly stronger limits than BaBar. Projections for mono-photon searches based on the same guiding principles as in BaBar and based on a fast data acquisition of $20 \mathrm{fb}^{-1}$ and a possible final experimental dataset of $50 \mathrm{ab}^{-1}$ have been given in refs. $[14,43]$. We have recasted the projections by adopting the same approach used for BaBar. We estimated the expected number of events for both the dark photon and the ALP, including the following experimental cuts (in the CoM frame):

$$
-0.85<\cos \theta_{\gamma}<0.91,
$$

where we used the boost factor from laboratory to CoM frame at Belle-II of 0.28 (compared to 0.56 for BaBar) and the angular coverage of the triggers from ref. [43]. Similarly to the BaBar case we do not include directly the energy cuts since their effect is mostly included in the limits shown in [43]. Interestingly, due to the larger angular coverage, the kinematics differences between ALP-related $s$-channel process and the dark photon one are somewhat larger than for BaBar (with dark photon distributions strongly localised around the upper limit for the mono-photon energy). This implies that an accurate estimate of Belle-II limits on $g_{a \gamma}$ would in fact require a dedicated simulation. Note however, that our projection still agrees very well with the estimate of the Belle-II reach performed in ref. [14]. The same issue does not arise in estimating the Belle-II reach for $g_{a e}$, as in this case the signal broadly shares the same characteristics than the dark photon signal.

\subsection{Limits from electron beam dump experiments}

Let us now focus on the limits arising from past and current electron beam dump experiments. Standard searches typically assume the ALP to decay visibly, and we thus expect limits from past electron beam dumps such as from the SLAC E141 [89] or E137 [44] experiments to be weakened strongly. In the following we will describe quantitatively this 
weakening by studying the E137 limits as function of the ALP visible branching ratio. Conversely, searches at NA64 [42] do not always rely on signals from visible decays, and will accordingly lead to stronger constraints.

E137. The SLAC E137 [44] experiment searched for light ALPs using a $20 \mathrm{GeV}$ electron beam dumped into aluminium (later iron) plates interlaced with cooling water. The shielding was provided from a neighbouring hill, corresponding to $D=179 \mathrm{~m}$ of shielding, and was followed by $L=204 \mathrm{~m}$ of open air decay region. The electromagnetic calorimeter was $2 \mathrm{~m} \times 3 \mathrm{~m}(3 \mathrm{~m} \times 3 \mathrm{~m})$ for Run 1 (Run 2$)$ and could detect charged particles and/or photons. The experiment accumulated a total dataset of $1.86 \times 10^{20}$ electrons on target (EoT), one third during the first run (with $\sim 10 \mathrm{C}$ of total electron charge) and the rest during Run $2(\sim 20 \mathrm{C})$. No candidate events with an energy above $\sim 2 \mathrm{GeV}$ and satisfying the various angular and timing cuts were found. Although one event was observed with energy higher than $1 \mathrm{GeV}$, there is the possibility that it originated from a cosmic ray muon [90].

In presence of both $g_{a e}$ and $g_{a \gamma}$, the ALP will be predominantly produced by Primakoff effect, due to the suppression of the bremsstrahlung process shown in eq. (2.22). ${ }^{8}$ Hence in the following we will focus exclusively on this production mechanism. Since the E137 search assumed visible decays of a long-lived ALP, it is clear that the reported limits get significantly weakened in a scenario in which invisible decays dominate. In order to estimate this effect, we start by considering the recasting of ref. [14] which inferred an upper limit on the couplings a function of the ALP mass for short-lived ALPs, on the basis of the single, lower-limit given by the experimental collaboration [44].

The E137 search for a long-lived ALP excludes a region of ALP-photon couplings between a lower limit $g_{d}^{\text {E137 }}$ corresponding to a very long-lived ALP and an upper limit $g_{u}^{\mathrm{E} 137}$ corresponding to a short-lived ALP. Both limits are function of the mass $m_{a}$, and ALPs with couplings $g_{a \gamma} \in\left[g_{d}^{\mathrm{E} 137}, g_{u}^{\mathrm{E} 137}\right]$ are excluded since they would decay in front of the apparatus with experimentally excluded rates. $g_{d}^{\mathrm{E} 137}$ and $g_{u}^{\mathrm{E} 137}$ have been extracted from ref. [14] (see in particular figure 2). If additionally the ALP has a significant invisible branching ratio, the upper limit will be significantly modified, since the invisible decay channels contribute to reduce the ALP lifetime. On the other hand, the lower limit, corresponding to the case of very long-lived ALP, does not get modified. In order to estimate the new upper limit $g_{u}^{\text {new }}$ we solve

$$
\left(g_{u}^{\text {new }}\right)^{2} \mathcal{P}^{\text {new }}=\left(g_{u}^{\text {E137 }}\right)^{2} \mathcal{P}_{u}^{\text {old }},
$$

where $\mathcal{P}^{\text {new }}$ and $\mathcal{P}^{\text {old }}$ denote respectively the ALP decay probability with and without invisible decay channels, and are given by

$$
\begin{aligned}
\mathcal{P}^{\text {new }} & \equiv \exp \left(-\frac{(L+D) \Gamma_{\text {inv }}\left(g_{u}^{\text {new }}\right)}{c \hbar \gamma_{a}}\right) \frac{L \Gamma_{\text {vis }}\left(g_{u}^{\text {new }}\right)}{c \hbar \gamma_{a}} \\
\mathcal{P}^{\text {old }} & \equiv \exp \left(-\frac{D \Gamma_{\text {vis }}\left(g_{u}^{\mathrm{E} 137}\right)}{c \hbar \gamma_{a}}\right) .
\end{aligned}
$$

\footnotetext{
${ }^{8}$ Note that the presence of secondary positrons in the electromagnetic showers implies that resonant production of ALP can also occur, although with a reduced geometric acceptance [75].
} 
In these relations we have assumed that the detection and geometric efficiencies are solely a function of the ALP mass, we have approximated $\Gamma_{\text {tot }} \approx \Gamma_{\text {inv }}$ for decays in the shield of length $D$, and we have included the ALP boost factor $\gamma_{a}=E_{a} / m_{a}$.

The above formulas further uses the fact that: (1) if the ALP decays mainly invisibly, the visible decay probability is small enough to approximate the exponential term by $\sim L \Gamma_{\text {vis }}\left(g_{u}^{\text {new }}\right) ;(2)$ in the original case of a visibly decaying ALP, the decay probability for the upper limit only requires that the ALP has not decayed in the shielding. Indeed, since $L \gtrsim D$, the surviving ALPs will then decay in the decay volume with near $100 \%$ probability.

Altogether, it is clear that the only missing information in these expressions is the ALP boost factor, which depends on the ALP energy distribution after geometrical cuts are applied. An average value for $\gamma_{a}$ can in fact be extracted using the above relations and the upper and lower limits $g_{u}^{\mathrm{E} 137}$ and $g_{d}^{\mathrm{E} 137}$. We obtain:

$$
\hbar c\left\langle\gamma_{a}\right\rangle=D \Gamma_{\gamma \gamma}\left(g_{u}^{\mathrm{E} 137}\right)\left[\mathcal{W}\left(\frac{\left(g_{u}^{\mathrm{E} 137}\right)^{4} D}{\left(g_{d}^{\mathrm{E} 137}\right)^{4} L}\right)\right]^{-1}
$$

where we have used the Lambert $\mathcal{W}$ function, and $\Gamma_{\gamma \gamma}\left(g_{u}^{\mathrm{E} 137}\right)$ is the ALP decay width to di-photon evaluated for $g_{a \gamma}=g_{u}^{\mathrm{E} 137}$. For small ALP masses, we find $m_{a}\left\langle\gamma_{a}\right\rangle \sim 15 \mathrm{GeV}$, in accordance with the naive expectation based on the fact that the E137 electron beam energy is $20 \mathrm{GeV} .{ }^{9}$ Replacing now in eqs. (3.12) and (3.13) $\gamma_{a}$ with $\left\langle\gamma_{a}\right\rangle$ and using eq. (3.11), we can readily find the new upper limit $g_{u}^{\text {new }}$.

Interestingly, the lower limit is not modified. This is because for both cases of dominant visible or invisible decays, it corresponds to the regime where the ALP is long-lived. Consequently, the number of expected visible decays simply scales with the visible width, as in the standard case. The above relations could also be applied to other beam dump limits on visible ALP decay (see, e.g. [19] for a recent update).

An important comment is that while the ALP may decay invisibly, it is clearly possible that its decay products still leave a signal in the detector. A typical example could be light dark matter scattering, such as in the case of the dark photon (note however, that the higher dimensional nature of the ALP portal tends to suppress this contribution w.r.t. the dark photon case). Another possibility is a three-body decay of some heavier dark sector state into lighter ones, as is typically found in inelastic dark matter scenarios. Since in this work we do not specify the structure of the dark sector, we will not include any of such limits. Finally, it has been pointed out recently that the inverse Primakoff process can also play a role to let light dark matter leave a signature in a detector [22].

NA64. NA64 is a fixed-target experiment which uses a $100 \mathrm{GeV}$ electron beam from the CERN SPS secondary beam line H4, and combines active beam dump and missing energy techniques to search for rare events. The experiment has accumulated $2.84 \times 10^{11}$ electronson-target (EoT) during the 2016-2018 run, and is expected to reach a total of $5 \times 10^{12}$

\footnotetext{
${ }^{9}$ Note that in all beam dump experiments, the upper limits are typically dominated by the fraction of events with the highest boost since, although for lower boosts one expects larger production rates, the number of detectable events gets exponential suppressed because of early decays.
} 
EoT. ALP production proceeds as in E137, with the Primakoff mechanism from secondary photon occurring directly within the electromagnetic calorimeter (ECAL). The ECAL is an active target assembled from lead and scandium plates that we model as 40 radiation lengths dump of lead. The NA64 search for ALPs [42] exploits two signatures: $a \rightarrow \gamma \gamma$ with an energy deposition $E_{\mathrm{ECAL}}<85 \mathrm{GeV}$, or large missing energy with $E_{\mathrm{ECAL}}<50 \mathrm{GeV}$. For our study the second signature is the relevant one, which implies that the ALP escaping ECAL needs to carry away at least $50 \mathrm{GeV}$ of missing energy. No events were observed by the collaboration [42], in agreement with an expected background of around 0.19 \pm 0.07 .

We have described in section 2.3 a simple approximation to the ALP production rate that applies to the NA64 setup. Note that the requirement that the ALP carries at least half of the initial electron energy implies that only events in which an ALP is produced in the first stage of the development of the electromagnetic shower in the ECAL will be relevant for this search. Including an experimental efficiency $\epsilon_{\mathrm{NA} 64} \simeq 50 \%$, we find that the expected number of events at NA64 is:

$$
N_{\mathrm{ALP}}=N_{\mathrm{EoT}} \epsilon_{\mathrm{NA} 64} \times \mathcal{N}_{A} \frac{\rho_{\mathrm{Pb}}}{A_{\mathrm{Pb}}} X_{0, \mathrm{~Pb}} \times\left[\left(\frac{g_{a e}}{1 \mathrm{GeV}^{-1}}\right)^{2} \sigma_{a e}^{\mathrm{eff}}+\left(\frac{g_{a \gamma}}{1 \mathrm{GeV}^{-1}}\right)^{2} \sigma_{a \gamma}^{\mathrm{eff}}\right],
$$

where $N_{\mathrm{EoT}}$ is the number of electrons on target, $\rho_{\mathrm{Pb}}=11.4 \mathrm{~g} / \mathrm{cm}^{3}$ is the lead mass density, $A_{\mathrm{Pb}}=207 \mathrm{~g} /$ mole the atomic mass, $X_{0, \mathrm{~Pb}}=0.56 \mathrm{~cm}$ the radiation length, and the effective cross-sections $\sigma_{a e}^{\text {eff }}$ and $\sigma_{a \gamma}^{\text {eff }}$, defined according to eq. (2.25), include the track-lengths integration as detailed in eq. (2.26). Note that the effective cross-sections account for the interaction between the electrons and the target nuclei, so that they include factors of the atomic number $Z_{\mathrm{Pb}}=82$. For the reference cross-sections $\sigma_{a \gamma}^{\text {eff }}$ and $\sigma_{a e}^{\text {eff }}$ we fix the couplings at the values $g_{a \gamma}=1 \mathrm{GeV}^{-1}$ and $g_{a e}=1 \mathrm{GeV}^{-1}$, and then we use the explicit dependence on the couplings as given in eq. (3.15) to derive the limits. To estimate the cross sections we have used the numerical setup based on MadGraph5_aMC@NLO described in section 2.3. We have included directly at the MC truth-level the energy cut, requiring the ALP to carry away at least $50 \mathrm{GeV}$. We set the $90 \%$ C.L. limit at 2.3 ALP events deriving limits on $g_{a e}$ and $g_{a \gamma}$ as well as prospects for the full NA64 dataset of $5 \times 10^{12}$ EoT. Remarkably, for the case of an ALP that is only coupled to photons $\left(g_{a e}=0\right)$ and for low values of $m_{a}$, our estimate of the limit agrees within $5 \%$ with the complete result from the collaboration. ${ }^{10}$

\subsection{Simulation of ALP production in the PADME environment}

The PADME experiment [45] at LNF uses a positron beam from the DA $\Phi$ NE LINAC accelerator in fixed target configuration, using as active target $100 \mu \mathrm{m}$ of polycrystalline diamond. The beam energy can be varied in the range $200-550 \mathrm{MeV}$. The experiment aims to collect a sample of $N_{\text {poT }} \sim 4 \cdot 10^{13}$ positrons on target (poT) and has recently completed RUN II collecting $\sim 5 \cdot 10^{12}$ poT. The current luminosity is mostly limited by a low duty factor $\sim 10^{-5}$ corresponding to 49 bunches of $200 \mathrm{~ns}$ length per second, each containing $\sim 2.8 \cdot 10^{4}$ positrons. Increasing the number of positrons per bunch would be easily feasible,

\footnotetext{
${ }^{10}$ The low mass limit corresponds to the case when the visibly decaying ALP searched for by the collaboration is long-lived, and hence mimics our signature, see also ref. [91].
} 
but it would lead to an excessive pile-up in the experiment. Future experimental prospects include large improvements to the DA $\Phi$ NE beam serving the experiment, (POSEYDON proposal [47]), increasing the duty cycle in the $10^{-2}-10^{-1}$ range by spreading the bunches over $0.2-2 \mathrm{~ms}$ at the same frequency, which would allow to increase $N_{\text {poT }}$ to around $(4-40) \cdot 10^{16}$ while reducing pile-up in the experiment. The electromagnetic calorimeter (ECAL) is designed to measure the final photon 4-momentum. It is placed at $3.45 \mathrm{~m}$ from the active target and it has a diameter of $60 \mathrm{~cm}$ and consequently the basic cuts applied on the final-state are [92]:

- angular coverage: $\left(15 \lesssim \theta_{\gamma} \lesssim 80\right) \mathrm{mrad}$, with $\theta_{\gamma}$ the photon angle with respect to the beam direction in the laboratory frame;

- energy range for a reconstructed cluster in ECAL: $30 \mathrm{MeV} \lesssim E_{\gamma} \lesssim 500 \mathrm{MeV} \cdot{ }^{11}$

The experimental technique adopted by PADME relies on the measurement of the missing mass in the final state. Indeed, knowing the initial conditions and measuring the single-photon four-momentum $p_{\gamma}$, it is possible to measure $m_{a}^{2}$ as the square of the missing mass:

$$
M_{\text {miss }}^{2}=\left(p_{e^{+}}+p_{e^{-}}-p_{\gamma}\right)^{2} .
$$

The signal would then correspond to a peak at $M_{\text {miss }}^{2}=m_{a}^{2}$ over a smooth distribution from the background $e^{+} e^{-} \rightarrow \gamma \gamma(\gamma)$ and from bremsstrahlung. Figure 6 a shows the missing mass square distributions for different values of $m_{a}$ together with an estimate of the background based on GEANT4 MC simulation from ref. [45]. The expected number of background events is around 3800 through the selection of $10^{11}$ simulated events. For reproducing the missing mass distribution we have simulated $10^{5}$ events by CalcHEP and applied the PADME cut using Root. We have also accounted for the spatial resolution of $4 \mathrm{~mm}$ and the measured energy resolution of ECAL [92],

$$
\frac{\sigma_{E}}{E_{\gamma}}=\frac{2 \%}{\sqrt{E_{\gamma}[\mathrm{GeV}]}} \oplus \frac{0.03 \%}{E_{\gamma}[\mathrm{GeV}]} \oplus 1.1 \% .
$$

The missing mass resolution for an ALP candidate $\left(m_{X}=m_{a}\right)$ depicted in figure $6 \mathrm{~b}$ shows a good agreement with the PADME technical design report for a dark photon. Including selection cuts, the differential distribution for ALP events is - up to an overall normalisation factor - roughly equivalent to the one for a dark photon (similarly to the case of BaBar). This is illustrated in figure 7 , where we show the distribution of the photon emission angle w.r.t. to the beam direction $\theta_{\gamma}$, for the case of a dark photon and of an ALP (both for $s$-channel and associated production processes). We have explicitly checked that also for PADME the interference term between the $s$ - and $t$-channel production processes is negligible in the whole parameter range.

Altogether, we note that the higher ALP mass region is most affected by the cuts as the resonant behaviour of the associated (fermionic) channel, presenting a soft photon, is cut by the energy selection.

\footnotetext{
${ }^{11}$ Note that for the associated production of ALP $+\gamma$, the energy of the emitted photon is always below $500 \mathrm{MeV}$ for a beam energy of $550 \mathrm{MeV}$.
} 


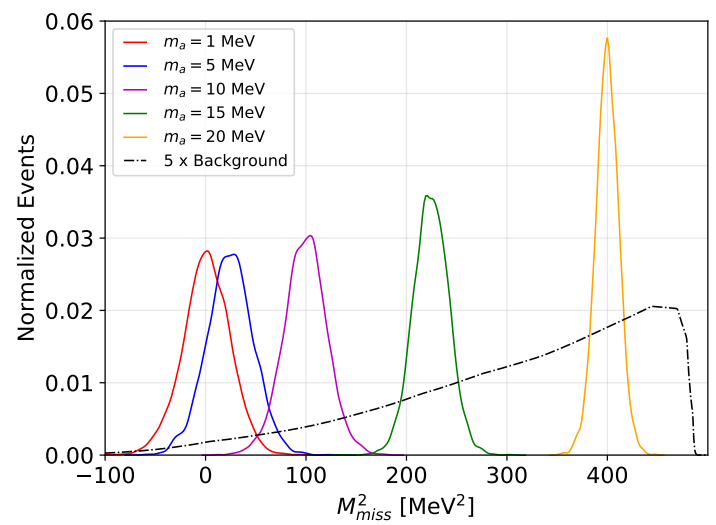

(a)

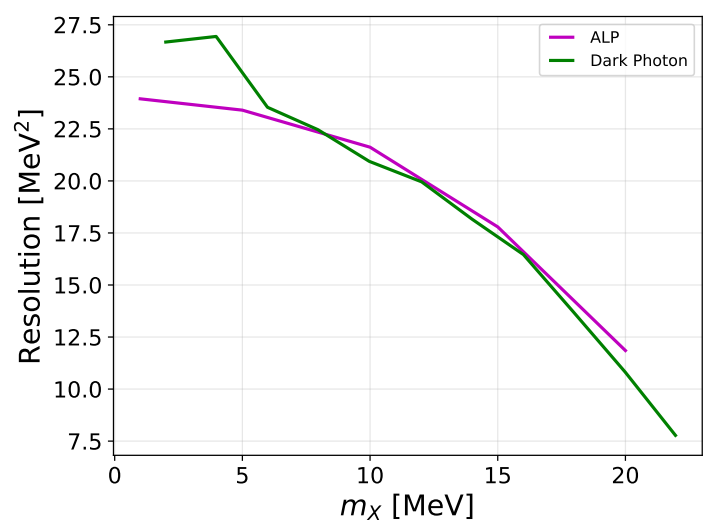

(b)

Figure 6. (a) Normalized $M_{\text {miss }}^{2}$ distribution for a set of ALP masses with PADME cuts applied, including energy and spatial resolution. The normalized distribution of background events (dasheddotted line) is extracted from [45]. (b) $M_{\text {miss }}^{2}$ resolution as a function of the dark particle mass $m_{X}$, where $X$ is an ALP or a dark photon, for $E_{\text {beam }}=550 \mathrm{MeV}$.
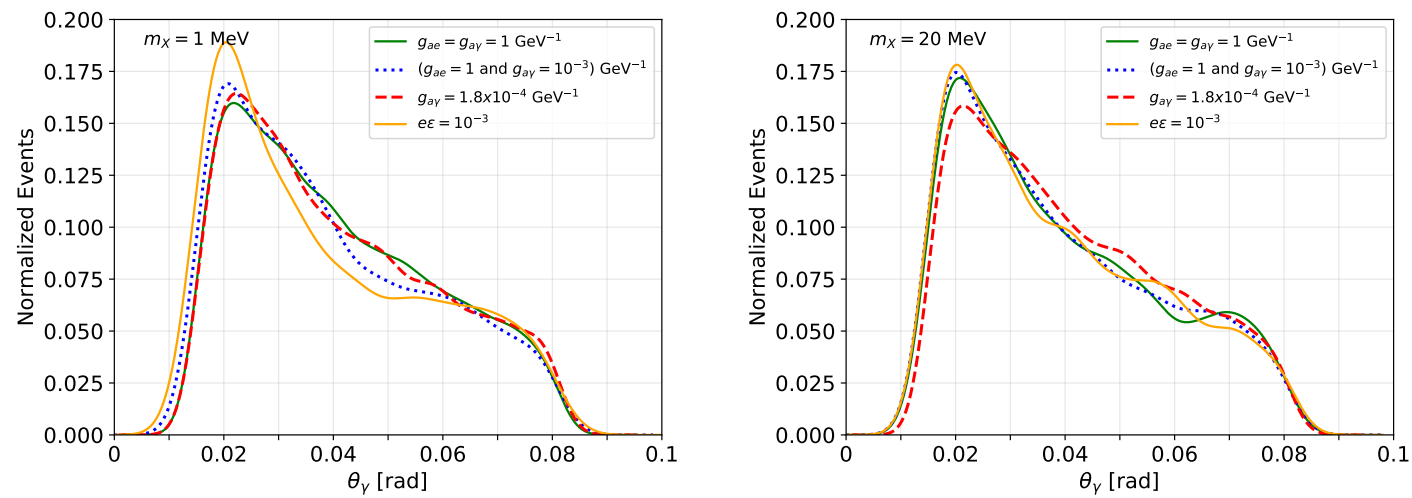

Figure 7. Normalized distribution for the angle $\theta_{\gamma}$ of a photon emitted in $e^{+} e^{-} \rightarrow \gamma X$, with $X$ a dark photon or an ALP, for $E_{\text {beam }}=550 \mathrm{MeV}$, and $m_{X}=1 \mathrm{MeV}$ (left), $m_{X}=10 \mathrm{MeV}$ (right). For the ALP the couplings are $g_{a e}=g_{a \gamma}=1 \mathrm{GeV}^{-1}$ (green line), $g_{a e}=10^{3} g_{a \gamma}=1 \mathrm{GeV}^{-1}$ (blue dotted line) and $g_{a e}=0, g_{a \gamma}=1.8 \cdot 10^{-4} \mathrm{GeV}^{-1}$ (red dashed line). For the dark photon (orange line) $e \epsilon=10^{-3}$.

In figure 8 , in the first panel we show the ALP production cross-section with and without cuts. We see that the contribution of the $t$-channel is heavily affected by the cuts in the large $m_{a}$ region. This is because the photon associated with the resonant regime is too soft and is cut out by the energy selection. In the low mass range the photon channel dominates. We see that for this channel the cuts do not reduce the signal by more than $50 \%$ in the whole mass range. In panel (b) we show the efficiency of the selection cuts for various values of the ratio $x=g_{a e} / g_{a \gamma}$. When the photon channel dominates over the fermion channel (dotted black line), the efficiency is about $60 \%$ in all the mass range up to $m_{a} \sim 21 \mathrm{MeV}$. When only the fermion channel is present (orange line), the efficiency is 


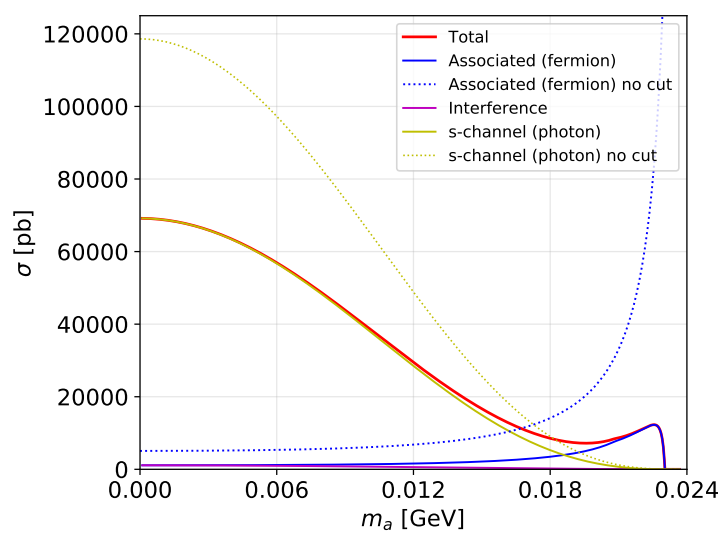

(a)

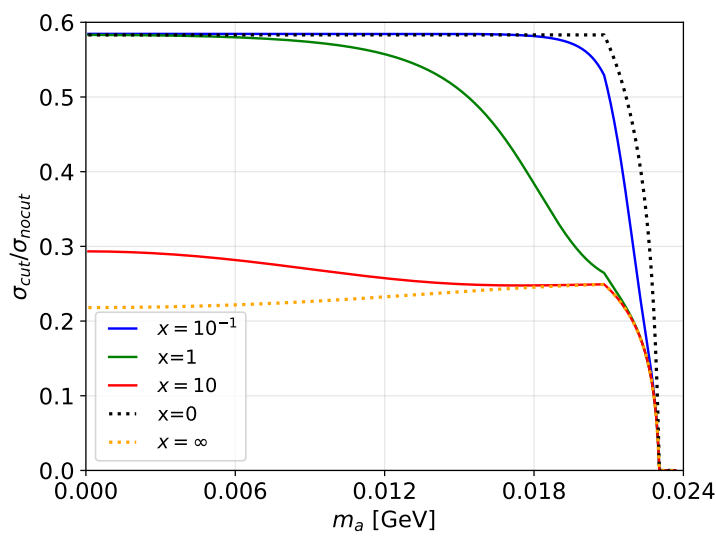

(b)

Figure 8. (a) Cross-section for $e^{+} e^{-} \rightarrow \gamma a$ as a function of $m_{a}$ for $g_{a e}=g_{a \gamma}=1 \mathrm{GeV}^{-1}$ and $E_{\text {beam }}=550 \mathrm{MeV}$ with the PADME cuts $15 \lesssim \theta_{\gamma} \lesssim 80 \mathrm{mrad}, 30 \mathrm{MeV} \lesssim E_{\gamma} \lesssim 500 \mathrm{MeV}$ and without cuts. (b) Selection efficiency for the total cross-section with the same cuts as a function of $m_{a}$ for different values of the ratio $x=g_{a e} / g_{a \gamma}$.

much smaller, around $25 \%$. For $m_{a} \gtrsim 21 \mathrm{MeV}$ the energy cut reduces the signal down to zero, as in this case the photon energy is always $\lesssim 30 \mathrm{MeV}$. From these results, it is clear that both annihilation channels are important to search for the $e^{+} e^{-} \rightarrow a \gamma$ signal. On the other hand, as the current limits on the $g_{a \gamma}$ limits are more stringent than on $g_{a e}$, PADME and its possible upgraded setups will be mostly relevant to probe the ALP coupling $g_{a e}$.

The expected number of events at PADME can be obtained from the total crosssection as

$$
N_{\mathrm{ALP}}=N_{\mathrm{poT}} \mathcal{N}_{A} \frac{Z_{C} \rho_{C}}{A_{C}} d_{t} \times\left(\varepsilon_{\mathrm{PA}} \sigma\right)_{\text {tot }}
$$

$\rho_{C}=3.5 \mathrm{~g} / \mathrm{cm}^{3}$ is the density of the diamond target, $A_{C}=12 \mathrm{~g} / \mathrm{mol}, Z_{C}=6, d_{t}=100 \mu \mathrm{m}$ is the target thickness, and $N_{\text {poT }}$ the number of positron on target. We have estimated the cross-section rescaled by the efficiency $\left(\varepsilon \sigma_{\text {tot }}\right)_{\mathrm{PA}}$ using CalcHEP and including the selection cuts for $g_{a e}=1 \mathrm{GeV}^{-1}$ and $g_{a \gamma}=1 \mathrm{GeV}^{-1}$ independently, which we denote respectively as $\left(\varepsilon_{\mathrm{PA}} \sigma\right)_{e}$ and $\left(\varepsilon_{\mathrm{PA}} \sigma\right)_{\gamma}$. We thus have:

$$
\left(\varepsilon_{\mathrm{PA}} \sigma\right)_{\mathrm{tot}}=\left(\frac{g_{a e}}{1 \mathrm{GeV}^{-1}}\right)^{2}\left(\varepsilon_{\mathrm{PA}} \sigma\right)_{e}+\left(\frac{g_{a \gamma}}{1 \mathrm{GeV}^{-1}}\right)^{2}\left(\varepsilon_{\mathrm{PA}} \sigma\right)_{\gamma}
$$

In order to estimate the background, we used the preliminary simulation described in [46]. The original aim of the collaboration was to perform a 'bump hunt' search in $M_{\text {miss }}^{2}$. As a conservative estimate, we have used the background events below the smeared resonance peak at $1.5 \sigma$ (corresponding to $85 \%$ of the signal). In estimating the future reach with the POSEYDON setup, we have assumed that detector improvements combined with reduced pile-up would lead to roughly the same number of background events, despite the strong increase in statistics. For the projection we will thus show a 100-signal events line. Since the production rate depends on the square of the couplings, the projected limits can be 


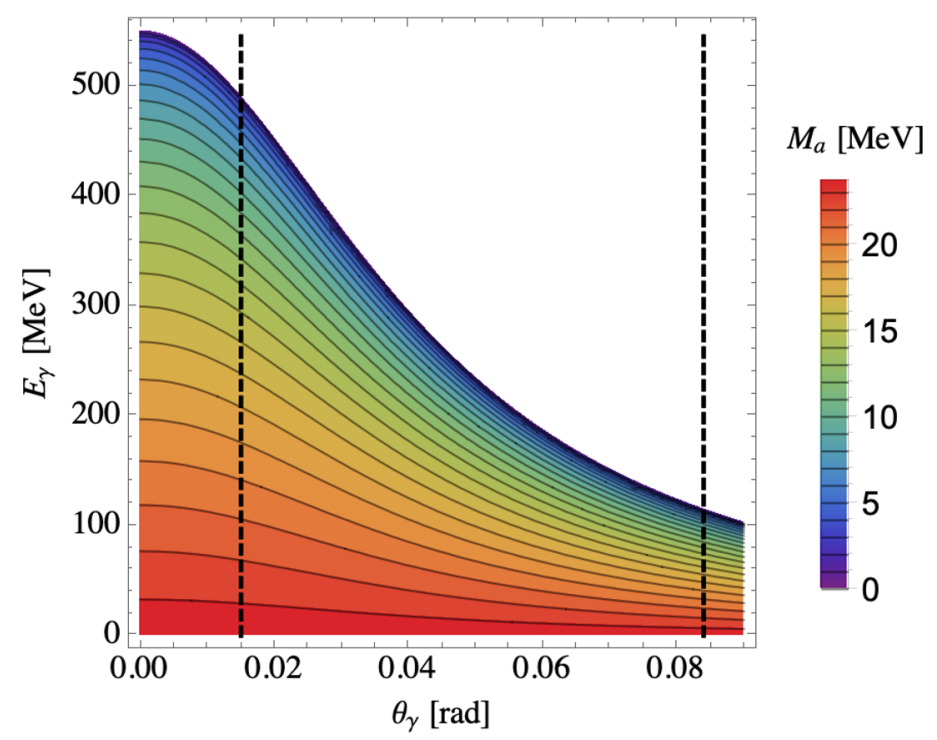

Figure 9. Correlation of the photon energy with its scattering angle at PADME. The gradient colour indicates the ALP mass. The dashed black lines are the angular separation between ECAL and SAC (Small Angle Calorimeter), and external ECAL border, respectively. The energy of the incident positron is $E_{\text {beam }}=550 \mathrm{MeV}$.

rescaled to the desired number of signal events $n_{s}$ by multiplying by $\sqrt{100 / n_{s}}$. To illustrate the maximum possible reach we further show the single event sensitivity (SES), corresponding to 2.3 signal events. It is interesting to speculate that a possible improved PADME experiment running with the POSEYDON setup with $\sim 10^{16}$ poT/year, while inheriting the same detection technique, might rely on a different detector and hence on different background rejection power. The actual limit will therefore depend on the performance of the new detector. In addition the forward region $\theta<15 \mathrm{mrad}$ as well as soft recoil photons, where most of the acceptance lies could be explored by a $10^{16}$ poT experiment, if the pile up could be reduced by factor of $\sim 100$ with respect to the current PADME setup.

Note that in case a signal is observed, the correlation between the photon energy and the emission angle can provide information about the ALP mass. Figure 9 shows how at small angles it is easier to distinguish different ALP masses, while at small $m_{a}$ the energy resolution of the calorimeter is crucial to pin down the precise value. The black dashed lines in the figure show the boundary of the ECAL region.

\subsection{Magnetic moment of light leptons}

The measured magnetic moment of the muon is one of the longest-standing anomalies in particle physics. Based on the original measurement at BNL [93] and on several improvements in the precision of the SM prediction (see e.g. the recent review [94]), the current discrepancy, parametrised in terms of $a_{\mu} \equiv(g-2)_{\mu} / 2$ is:

$$
\Delta a_{\mu} \equiv a_{\mu}^{\exp }-a_{\mu}^{\mathrm{SM}}=(2.79 \pm 0.76) \cdot 10^{-9}
$$




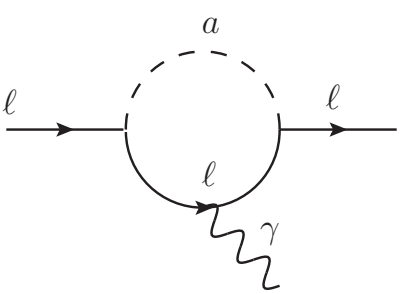

(a)

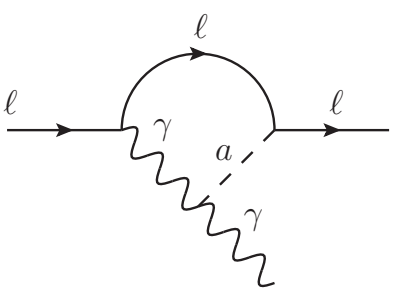

(b)

Figure 10. Feynman diagrams for the two main contributions of an ALP to $\Delta a_{e}$ and $\Delta a_{\mu}$. Diagram (a) is proportional to $g_{\ell}^{2}$, while the Barr-Zee diagram in (b) is proportional to $g_{a \gamma} g_{\ell}$.

corresponding to a $3.7 \sigma$ anomaly. Intriguingly another anomaly was recently observed also in the anomalous magnetic moment of the electron. Comparing the improved measurement of the fine structure constant from the study of cesium-133 atoms in a matter-wave interferometer [95] with the SM theoretical prediction [96] yields:

$$
\Delta a_{e} \equiv a_{e}^{\exp }-a_{e}^{\mathrm{SM}}=-(8.7 \pm 3.6) \cdot 10^{-13} \quad(\text { Berkeley-2018) }
$$

corresponding to a $2.4 \sigma$ tension. Although the observation of two discrepancies in the analogous observable for the muons and the electrons is indeed suggestive, the fact that the two deviations have discording signs poses a significant theoretical challenge for finding an explanation in terms of new physics. Loop contributions from new light vector or scalar particles typically lead to a positive contribution to both the theoretical predictions for $a_{e}^{\text {th }}$ and $a_{\mu}^{\text {th }}$, while contributions from axial-vector or pseudo-scalar particles are instead negative. As the corrections are typically proportional to the square of the couplings of the new particles, one would expect that both deviations come with the same sign. An ALP, that is a light pseudo-scalar, can provide one of the most elegant solution to explain both anomalies in one go, due to the simultaneous presence of the photon and the electron/muon coupling. The diagrams that yield the main contributions are drawn in figure 10. Crucially, the second diagram in figure (b) is proportional to $g_{a \gamma} g_{a \ell}$ and can therefore change sign depending on the sign of the ALP coupling to the particular fermion involved [25, 97]. Summing also the contribution of diagram (a) (see, e.g. [28]) as well as the 2-loop contribution from a light-by-light diagram [25] leads to the overall correction:

$$
a_{\ell}^{\mathrm{th}}-a_{\ell}^{\mathrm{SM}}=\frac{m_{\ell}^{2}}{16 \pi^{2}}\left(2 g_{a \gamma} g_{a e}\left(h_{2}-\log \frac{\Lambda^{2}}{m_{\ell}^{2}}\right)-g_{a e}^{2} h_{1}+g_{a \gamma}^{2} \frac{3 \alpha_{\mathrm{em}}}{\pi} \log ^{2} \frac{\Lambda}{m_{\ell}}\right),
$$

where the loop functions $h_{1}(x)$ and $h_{2}(x)$, with $x=m_{a}^{2} / m_{\ell}^{2}$, are:

$$
\begin{aligned}
& h_{1}(x)=\int_{0}^{1} d z \frac{2 x^{3}}{x^{2}+z(1-x)} \\
& h_{2}(x)=1-\frac{x}{3}+\frac{x^{2}}{6} \log x+\frac{2+x}{3} \sqrt{x(4-x)} \arccos \frac{\sqrt{x}}{2} .
\end{aligned}
$$

The possibility of a simultaneous fit to both anomalies on the basis of eq. (3.21) has been thoroughly explored in the literature. The earlier attempts were based on a simplified model 
approach [26, 27], and later lepton-flavour violating interactions were also used [28, 29]. In the following, we focus on the flavour-diagonal case, which can explain both anomalies using only Barr-Zee diagrams, assuming a GeV-scale ALP and the products of couplings $g_{a \gamma} g_{a e} \sim 10^{-5} \mathrm{GeV}^{-2}$ and $g_{a \gamma} g_{a \mu} \sim-10^{-6} \mathrm{GeV}^{-2}$.

Several comments are in order. First, it is interesting to note that the contribution of $g_{a e}$ to the electromagnetic anomaly (see section 2), is precisely of the adequate order of magnitude to fit $\Delta a_{e}$ with $g_{a e} \sim(0.01-0.1) \mathrm{GeV}^{-1}$. Additionally, while the required couplings to the fermions are of significant size, that this does not imply per-se that the corresponding scale $\Lambda$ has to be very small. From a UV perspective, the ALP interactions arise proportionally to the charges of the approximate broken global symmetry. A large coupling to a fermion species may thus simply indicate that this species boasts a large value of the global charge. The above reasoning has been employed in various ways in the context of axion model building to boost selectively some axion couplings, see for instance [98]. We also note that such a UV-complete theory with non-universal ALP couplings to leptons will in general give rise to flavor-violating ALP-lepton interactions. We refer to [29, 99] for a thorough study of the constraints in this case. In the following, we will mostly focus on the case of the electron anomaly $\Delta a_{e}$, as we aim at constraining the ALP couplings to electrons and photons. We will nonetheless briefly comment also on the muon anomaly at the end of this work. As a final comment, it is clear that the contributions from eq. (3.21) do not include possible direct effects from the UV theory. Given that both anomalies are known to be strongly sensitive to the presence of new particles up to the $\mathrm{TeV}$ scale, it is advisable to consider these anomalies more as potential guidelines to identify some type of new physics, rather than using these measurements to constrain the ALP couplings on and equal footing than the direct searches at accelerators.

Before concluding this section, it should be mentioned that the most recent atomic physics measurement of $\alpha_{\mathrm{em}}$ using Rubidium-87 atoms [100] reported a value for $\alpha_{\mathrm{em}}$ that is more than $5 \sigma$ away from the previous result of [95] (and at $\sim 2 \sigma$ from an earlier Rubidium- 87 measurement). Taken at face value, this would imply

$$
\Delta a_{e} \equiv a_{e}^{\exp }-a_{e}^{\mathrm{SM}}=+(4.8 \pm 3.0) \cdot 10^{-13} \quad(\mathrm{LKB}-2020),
$$

still in mild tension with the SM but, most importantly, the deviation would now have a positive sign. Clearly, the $5 \sigma$ discordance between the two most precise experimental measurements calls for a clarification, and it would be highly desirable that an agreement on the values of $\alpha_{\mathrm{em}}$ could be reached in the forthcoming coming years. As regards our results, they can be straightforwardly adapted to either sign of the deviation, and this is because in this work $\Delta a_{e}$ is the only observable which carries a dependence on the sign of $g_{a e}$, and hence switching the sign of this coupling $g_{a e}$ allows to fit both possibilities. In the following we will use the results from eq. $(3.20) .{ }^{12}$

\footnotetext{
${ }^{12}$ We have checked numerically that the conclusions derived in the next section remain unchanged had we instead used eq. (3.24). The only exception is that there is no parameter space region compatible with $\Delta a_{e}$ in the pure electrophilic case $\left(g_{a \gamma}=0\right)$ shown in figure 12. However, even the region compatible with eq. (3.20) is already almost entirely excluded by other measurements.
} 


\section{Results}

Relying on the analysis of the previous sections, we are now in the position to discuss the updated status of searches for ALPs decaying (semi)-invisibly. In the plots of this section we will also underline when the relevant parameters fall in a range that could also explain the deviations from the SM predictions for the values of the $\mu$ and $e$ anomalous magnetic moments.

\subsection{Photo-philic or electro-philic ALP}

Let us first consider the case of a photo-philic ALP, that is an ALP for which the coupling $g_{a \gamma}$ is the dominant one. The corresponding limits are presented in figure 11. From this plot it is clear that the mono-photon search from BaBar [41] dominates the constraints in most of the parameter space. In particular, and in contrast with the case of a dark photon or of a visibly decaying ALP, the NA64 missing energy search [42] is so far providing subdominant constraints. Nonetheless, with the full $5 \cdot 10^{12}$ EoT dataset, NA64 could eventually take over BaBar, and compete with the Belle-II reach based on a first dataset of $20 \mathrm{fb}^{-1}$. In the long run, the results from Belle-II will likely dominate the limits by about an order of magnitude. Limits from PADME do not lead to new exclusion regions and are not shown in the plot.

Interestingly, the limits from visible searches in beam dump experiments, such as E137, can remain relevant even when the ALP visible decay branching ratio is largely suppressed. This is shown in figure 11: even with a visible branching ratio $\mathrm{BR}_{\mathrm{vis}}^{\mathrm{ALP}} \equiv\left(\Gamma_{\gamma \gamma}+\Gamma_{\mathrm{e}^{+} \mathrm{e}^{-}}\right) / \Gamma_{\mathrm{inv}}$ as small as $10^{-4}$ (dot-dashed grey line), the E137 limit remains relevant in the low mass region, due to the fact that in this region the BaBar and NA64 limits saturate. This shows that for ALPs decaying partially invisibly, all beam dump limits need to be estimated carefully. Note that we do not show the limit from $\Delta a_{e}$, since the strong constraints on the photon coupling imply that the light-by-light contribution from eq. (3.21) is negligible (besides having the wrong sign to accommodate the measurement of $\Delta a_{e}$ ).

In figure 12 we present the case of a purely electrophilic ALP $\left(g_{a \gamma}=0\right)$. Due to the scaling of the electron bremsstrahlung cross-section with the electron-ALP mass ratio (see eq. (2.22)) the limits from electron beam dump, and in particular from NA64, get stronger with decreasing $m_{a}$. In line with the general scaling argument presented in section 2 , the limits follow the same hierarchy as for the better known case of a dark photon portal. NA64 dominates the limits in the lower mass region (below around $200 \mathrm{MeV}$ ), while BaBar and soon-to-be release results from Belle-II provide the strongest constraints up to around $10 \mathrm{GeV}$. Probing higher ALP masses is significantly harder for intensity frontier experiments, so that LEP mono-photon searches still provide the strongest bounds in the tens of $\mathrm{GeV}$ range. It is worth noting that in this region the 'pure' $g_{a e}$ contribution to $\Delta a_{e}$ could still accommodate the experimental measurement, although most of the useful parameter space is already excluded. With an upgrade of the experimental setup, in a five-year timescale PADME could probe part of the parameter space accessible at NA64 only after the full expected dataset will be collected. 


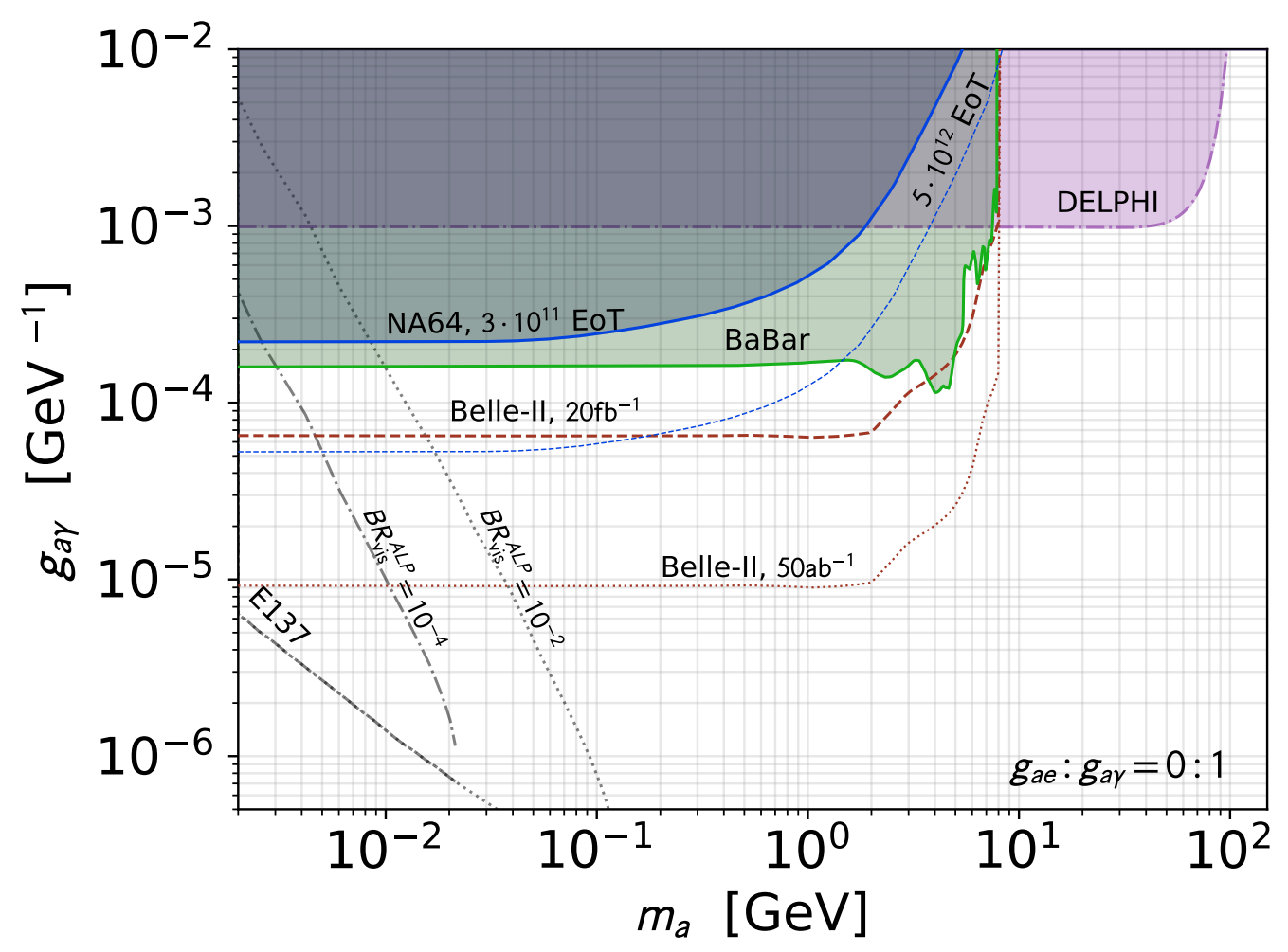

Figure 11. Summary of limits for an ALP decaying preeminently invisibly, when $g_{a \gamma}$ dominates over $g_{a e}$. The dotted and dash-dotted grey lines correspond to the range excluded by E137 [44] for visible branching ratio respectively of $10^{-2}$ and $10^{-4}$. The purple region is the exclusion from the DELPHI mono-photon search [40, 84]. The current (future) limits from NA64 [42] correspond to the solid (dotted) blue line. The green region is the exclusion from mono-photon search at BaBar [41]. The dashed (dotted) rust line represent the projected reach of Belle-II [43] for a $20 \mathrm{fb}^{-1}$ $\left(50 \mathrm{ab}^{-1}\right)$ dataset.

\subsection{Combined case}

Let us now turn to the case where both couplings are present and they are both relevant. We show the limits for this case in the three panels of figure 13 , for $m_{a}=0.01,1$ and $7.5 \mathrm{GeV}$. In most cases the presence of both couplings does not lead to a striking new behaviour. For instance, for NA64, PADME, BaBar and Belle-II (projective) limits, a smooth transition is observed when both the electron and photon contributions to the ALP production rates are of similar size. In the case of the E137 limits, note that although we have only included ALP production via the Primakoff mechanism, the electron channel is the main visible ALP decay mode in the right-hand part of the three plots. A remark is in order for the surviving limit that can be obtained from beam dump experiments for $m_{a}$ in the few $\mathrm{MeV}$ range. This is illustrated by the E137 excluded region shown as the grey area in figure 13a, which corresponds to an ALP visible branching ratio of $10^{-4}$. The assumption that the ALP dominantly decays invisibly, implies that for $m_{a}=10 \mathrm{MeV}$ and $g_{a e} \gtrsim 5 \cdot 10^{-5} \mathrm{GeV}^{-1}$ the invisible decay length becomes much smaller than the length of the shielding, so that 


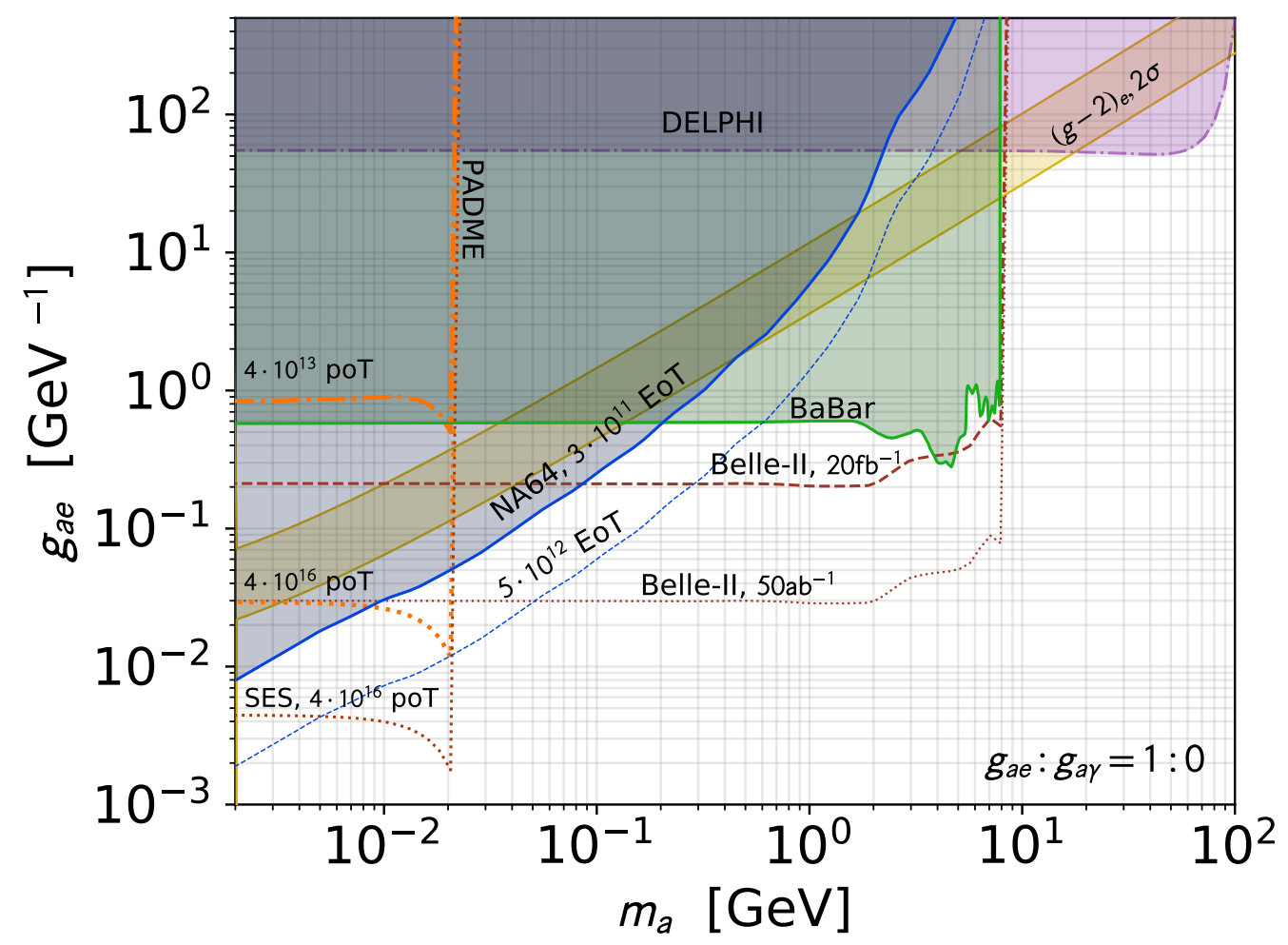

Figure 12. Summary of the limits when $g_{a e}$ dominates over $g_{a \gamma}$. The three broken orange lines are (top to bottom) the projection for PADME [46] for the current run, and for a POSEYDON [47] upgrade of the beam with either 100 signal events, or with single-event sensitivity (SES - 2.3 events line). The purple region is the exclusion from DELPHI mono-photon searches [40, 84]. The solid (dotted) blue line corresponds to the current (future) limit from NA64 [42]. The green region is the exclusion from mono-photon searches at BaBar [41]. The dashed (dotted) rust line gives the prospects at Belle-II [43] for a $20 \mathrm{fb}^{-1}\left(50 \mathrm{ab}^{-1}\right)$ dataset. The golden strip corresponds to the $2 \sigma$ range for the $(g-2)_{e}$ anomaly.

there is no bound beyond this point. The second observable which depends non-trivially on both couplings is the correction to the electron anomalous magnetic moment $\Delta a_{e}$. The combination of the pure $g_{a e}^{2}$ contribution and of the Barr-Zee $g_{a e} g_{a \gamma}$ term opens up an interesting region in parameter space, that remains below the current NA64 and BaBar limits, for ALP masses larger than about $10 \mathrm{MeV}$. The experimental prospects to probe this region are rather good, hence the $(g-2)_{e}$ anomaly constitutes an obvious target for future improved limits from NA64, PADME and Belle-II. The $2 \sigma$ region wherein the discrepancy can be explained (shown as the gold strip in the three figures) could be likely covered almost entirely, in particular thanks to future Belle-II limits.

Finally, the interplay between the limits from NA64 and BaBar is of interest. The former is relevant only for low ALP masses, while the latter provides significant constraints also for $m_{a} \gtrsim 1 \mathrm{GeV}$. Moreover, it can be seen from figure 13a that for $m_{a} \sim 10 \mathrm{MeV} \mathrm{BaBar}$ dominates the limits on $g_{a \gamma}$, while NA64 provides the strongest constraints on $g_{a e}$. This 


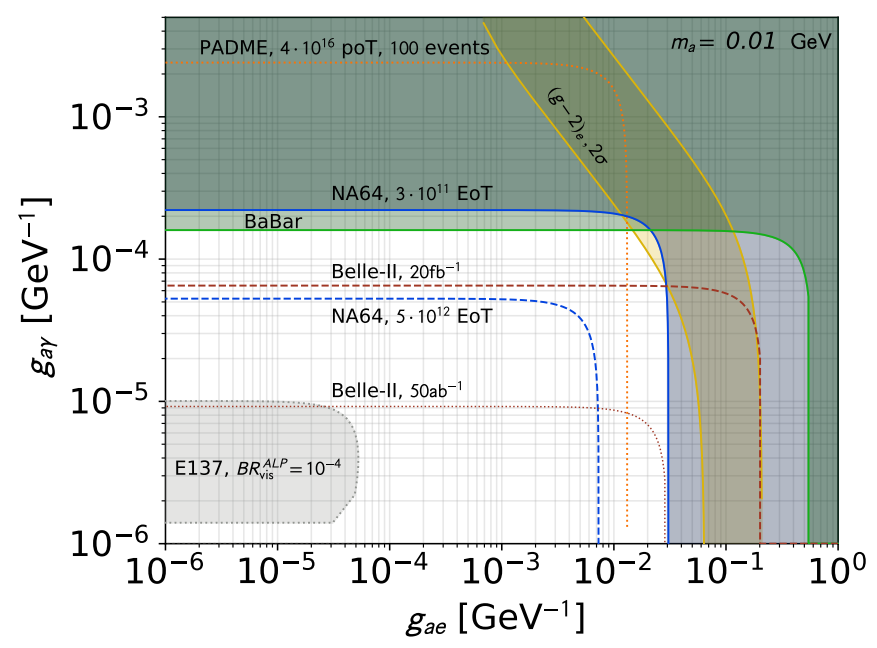

(a)

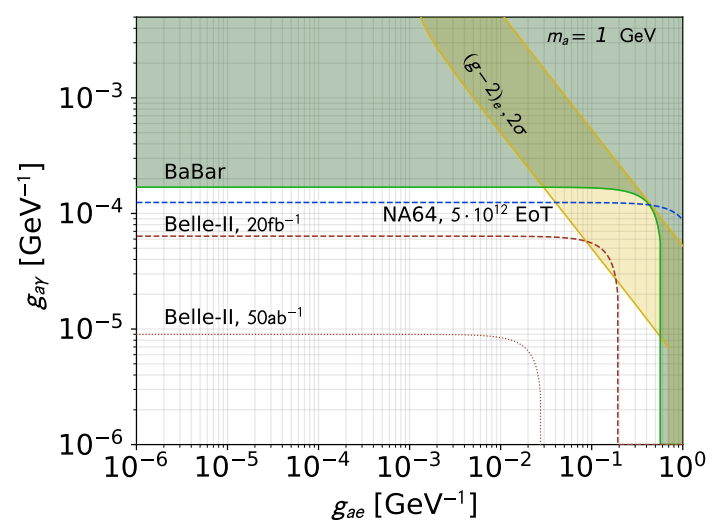

(b)

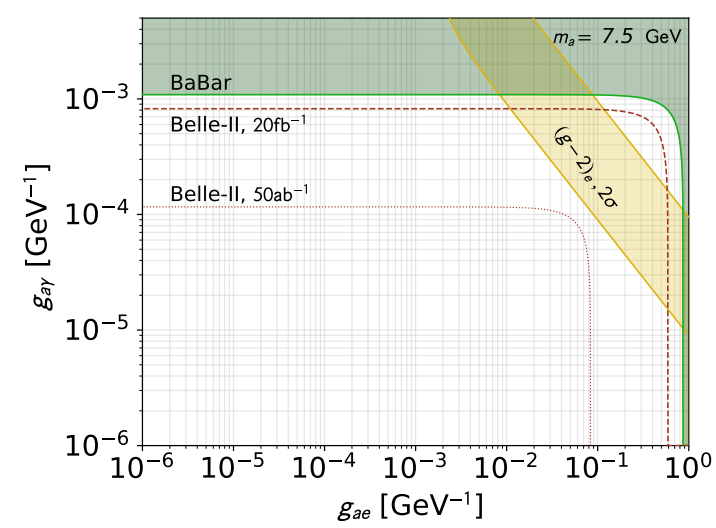

(c)

Figure 13. Summary of limits as function of $g_{a e}$ and $g_{a \gamma}$ for $m_{a}=10 \mathrm{MeV}$ (13a), $m_{a}=1 \mathrm{GeV}(13 \mathrm{~b})$ and $m_{a}=7.5 \mathrm{GeV}$ (13c). The grey region in 13a) is excluded by E137 [44] assuming a visible branching ratio of $10^{-4}$. The dashed orange lines is the projection for a POSEYDON [47] extension of PADME with 100 signal events. The purple region is the exclusion from the DELPHI monophoton search [40, 84]. The current (future) limits from NA64 [42] corresponds to the solid (dashed) blue line. The green region is the exclusion from mono-photon search at BaBar [41]. The dashed (dotted) rust line represents the prospects at Belle-II [43] for a $20 \mathrm{fb}^{-1}\left(50 \mathrm{ab}^{-1}\right)$ dataset. The golden strip corresponds to the $2 \sigma$ range for the $(g-2)_{e}$ anomaly.

can be traced back in large part to the $1 / s$ suppression of the associated cross-section in electron-positron colliders.

We investigate further the mass dependence of these limits in figure 14 . We show the limits on the ALP couplings as function of $m_{a}$ for $g_{a e} / g_{a \gamma}=10^{3}$ in figure $14 \mathrm{a}$ and for $g_{a e} / g_{a \gamma}=10^{2}$ in figure 14a. The first choice corresponds roughly to the expected size of $g_{a \gamma}$ when is generated radiatively from an electron loop. In both cases, the limits are typically dominated by the photon-mediated processes, particularly for BaBar and Belle-II, while 


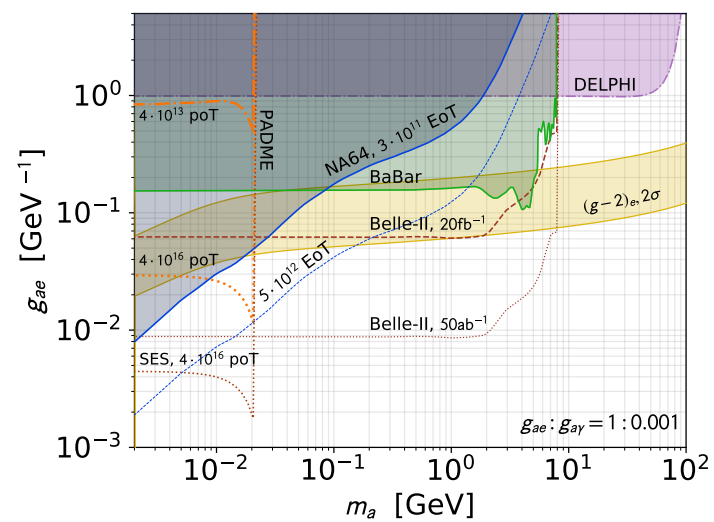

(a)

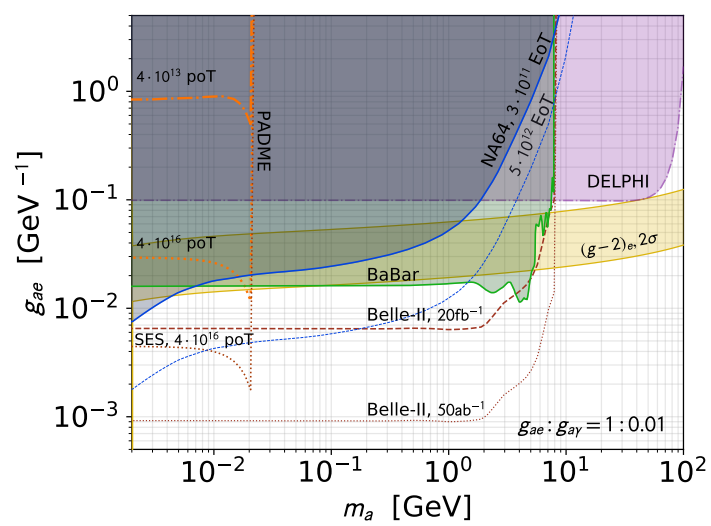

(b)

Figure 14. Summary of limits as function of $g_{a e}$ and $m_{a}$ for $g_{a e} / g_{a \gamma}=10^{3}(14 \mathrm{a})$ and $g_{a e} / g_{a \gamma}=$ $10^{2}$ (14b). A negligible ALP visible branching ratio is assumed. The dashed orange lines are (top to bottom) the projection for PADME [46] for the current run, and for a POSEYDON [47] extension with either 100 signal events, or with single-event sensitivity (SES - 2.3 events line). The purple region is the exclusion from DELPHI mono-photon searches [40, 84]. The current (future) limits from NA64 [42] correspond to the solid (dotted) blue line. The green region is the exclusion from BaBar [41]. The dashed (dotted) rust line gives the projection for Belle-II [43] for a $20 \mathrm{fb}^{-1}$ $\left(50 \mathrm{ab}^{-1}\right)$ dataset. The golden strip corresponds to the $2 \sigma$ range for the $(g-2)_{e}$ anomaly.

for NA64 for large values of $m_{a}$ the limits are determined by $g_{a \gamma}$, but become dominated by $g_{a e}$ as the ALP mass is decreased. The figure also illustrate the large parameter space available for an electrophilic ALP $\left(g_{a e} \gg g_{a \gamma}\right)$ solution to the $\Delta a_{e}$ anomaly, with the viable parameter space extending down to tens of $\mathrm{MeV}$. In the absence of any significant compensating UV contribution, one can regard the area above the golden strip as excluded by the $\Delta a_{e}$ measurement. However, by construction the ALP Lagrangian eq. (2.1) defines a low energy effective theory, which assumes the presence of an UV sector with a larger field content. One should therefore be cautious regarding limits derived from $(g-2)_{e}$ because additional contributions to from the UV sector to this quantity are certainly possible.

We conclude this section by extending the ALP interactions to include also an interaction with the muon controlled by the coupling $g_{a \mu}$. This renders possible to fit simultaneously both the $\Delta a_{e}$ and $\Delta a_{\mu}$ anomalies. Figure 15 shows the preferred region in the $\left(m_{a}, g_{a e}\right)$ plane for a ratio of the fermion couplings $g_{a e} / g_{a \mu} \sim-10$. As expected from previous results (see e.g. [29]) the choice of this value for the ratio of the lepton couplings provides a very good fit in the range of large ALP masses. However, we find that the combined region extends to mass values much lower than what was previously though, as the BaBar results are in fact not sufficient to exclude the $\Delta a_{\mu}$-favoured region below $10 \mathrm{GeV}$. Moreover, it is interesting to remark that the prediction from a radiatively generated ALP-photon coupling $g_{a \gamma} / g_{a e} \sim 10^{-3}$ leads to an adequate range for the photon interaction. Indeed this ratio offers a good compromise between a suppression of accelerator and collider limits (in particular from BaBar) and a sufficiently large enhancement of both $\Delta a_{e}$ and $\Delta a_{\mu}$ via the Barr-Zee contribution. Note that we have not included limits 


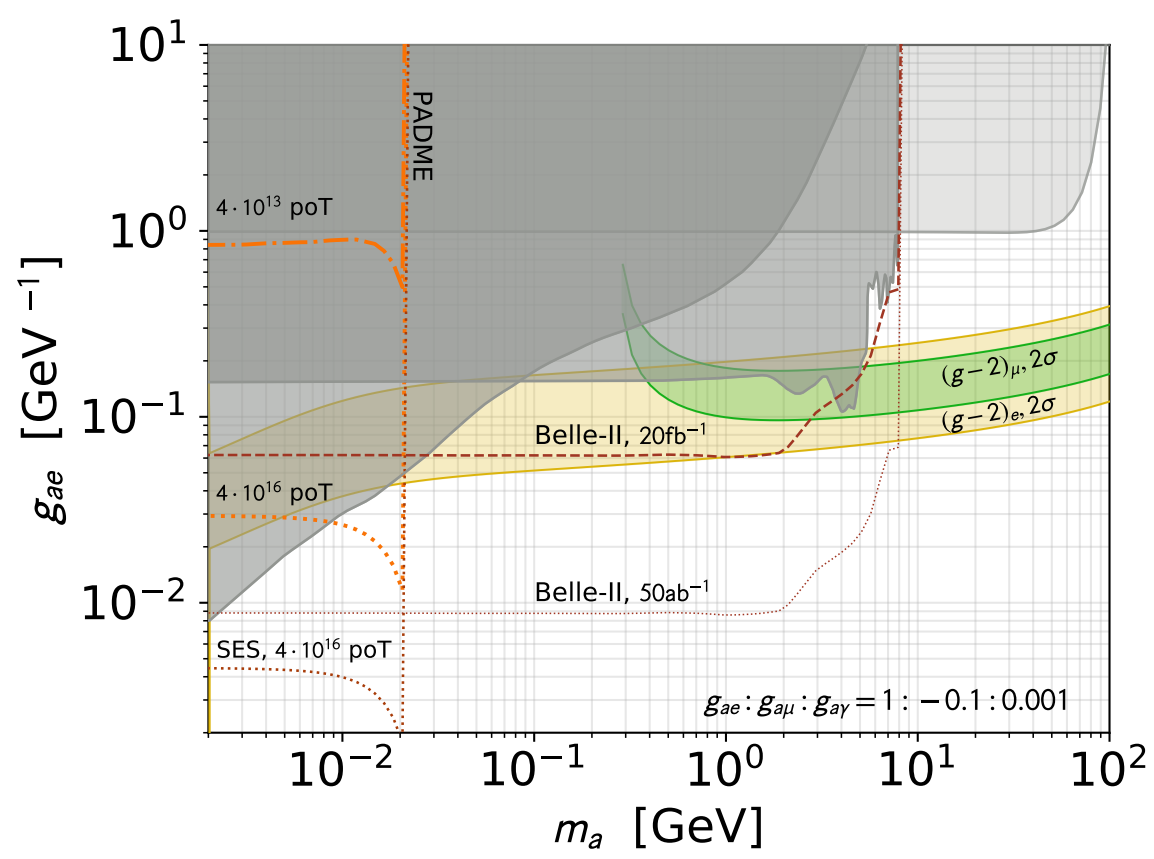

Figure 15. The invisible ALP $(g-2)_{e, \mu}$ solution. Summary of limits for $g_{a e} / g_{a \gamma}=10^{3}$ and $g_{a \mu} / g_{a \gamma}=-10^{2}$. The dashed orange lines (from top to bottom) give the projection for PADME [46] for the current run, and for a POSEYDON [47] extension with either 100 signal events, or with single-event sensitivity (SES - 2.3 events line). The grey regions are exclude by DELPHI [40, 84], NA64 [42] and BaBar [41]. The dashed (dotted) rust line shows the projected limits from BelleII [43] for a $20 \mathrm{fb}^{-1}\left(50 \mathrm{ab}^{-1}\right)$ dataset. The golden strip corresponds to the $2 \sigma$ range for explaining the $(g-2)_{e}$ anomaly, and the green region to the $2 \sigma$ range for the $(g-2)_{\mu}$ anomaly.

on $g_{a \mu}$ from the recast of past beam dump experiments (e.g. E137 limits [101]) or projected limits from possible future experiments such as NA64- $\mu$ [102], $M^{3}$ [103] or from dedicated Belle-II searches [104]. This is because the fit requires an ALP muon coupling sizeably smaller than the electron one, hence we expect that future limits on $g_{a e}$ will keep providing the strongest constraints for the ALP solution to the $g-2)_{e, \mu}$ anomalies.

\section{Conclusions}

In this work we have presented a thorough analysis of the experimental constraints on the scenario in which an ALP constitutes a portal to a dark sector, and dominantly decays into light dark particles, while in the SM sector it couples to photons and electrons to which it can also decay, but with subdominant branching ratios. We have first reviewed existing astrophysical constraints on this scenario, questioning in particular the limits from the duration of the SN1987 neutrino burst, which rely on the fact that the burst duration would be shortened if the ALP decay products could freely escape from the proto-neutron star core. We have in fact shown that interactions within the dark sector of a reasonable strength may suffice to keep the light dark particles trapped inside the core, along with the ALPs, on the same time scale of neutrino trapping. We have then proceeded to perform a 
thorough re-interpretation of all the relevant collider and beam dump experiments relevant to constrain this scenario. Such a study was not yet available for the case of an ALP decaying dominantly into invisible channels. Not surprisingly, we have shown that the stronger constraints arise from various missing energy searches in BaBar and NA64, and from and mono-photon searches at LEP, and in particular from the results reported by the DELPHI collaboration [40]. Although the DELPHI mono-photon search results have been already widely used to constraint both an invisible dark photon and an invisible ALP, we have accounted for the first time for the variation of the LEP center-of-mass energy during the run time. This has the important effect of shifting the mass threshold of the analysis to larger ALP masses. We have reinterpreted NA64 limits on visible ALP decays in terms of limits on invisible decay channels, mimicking to a certain extend the results found by the collaboration for the case of an invisible dark photon. We have also shown that relaxing completely the limits from existing searches for long-lived ALPs is challenging, and requires a significant suppression of the visible branching ratios. We have illustrated this point for the particular case of the SLAC E137 beam dump experiment, however, the procedure that we have described allows in principle to recast in the same way the results of other beam-dump based searches for long-lived ALPs. Finally we have investigated the reach of the current run of the PADME positron beam dump experiment, and we have discussed the projected sensitivity in case the positron beam delivered by the LNF Beam Test Facility could be upgraded according to the POSEYDON proposal.

An invisible ALP as the one investigated in this work is among the preferred new physics candidates for explaining the discrepancy between the predicted value of the anomalous magnetic moment of the electron and the result of a recent measurement. We have confronted the parameter range hinted at by this discrepancy with the limits we have derived on the ALP mass and couplings, finding that a sizeable parameter space region is still viable, which thus constitutes an important target for upcoming experiments. In particular, we have argued that both the NA64 and Belle-II experiments will be able to probe in the near future a large part of this region for an ALP mass below $10 \mathrm{GeV}$. We have additionally discussed the possibility of a simultaneous explanation for the deviations of the $(g-2)_{e}$ and $(g-2)_{\mu}$ measured values from the SM predictions. We have shown that if the ALP couples to the muon with a coupling about a factor of ten smaller than the electron coupling, and of opposite sign, the ALP explanation is viable for ALP masses as low as a few hundreds of $\mathrm{MeV}$. This observation provides an additional golden target for future experimental studies.

Note added. Shortly after this paper first appeared, the result of a new experimental measurement of the muon anomalous magnetic moment by the FNAL Muon g-2 experiment was published [105], that when combined with the old BNL result [93] yields the new world average

$$
\Delta a_{\mu} \equiv a_{\mu}^{\exp }-a_{\mu}^{\mathrm{SM}}=(2.51 \pm 0.59) \cdot 10^{-9} .
$$

While this new measurement increases the tension with the SM prediction at the level of $4.2 \sigma$, it does not significantly modify the central value. Thus our results, and in particular figure 15 , remain compatible with the new experimental value. 


\section{Acknowledgments}

We acknowledge discussions with L. Delle Rose and R. Simeonov at the beginning of the project, P. Valente for providing us with the details of the DA $\Phi$ NE POSEDYON setup, and G. Corcella for important advice on the numerical aspects of this work. We thank P. Paradisi for useful comments on the first version of the paper, and M. Hirsch for bringing to our attention ref. [100]. L.D. thanks F. Kahlhoefer for useful feedback on ALP astrophysics. L.D. and E.N. acknowledge the PADME collaboration for fundamental inputs on the experimental analysis. L.D. and E.N. are supported in part by the INFN "Iniziativa Specifica" Theoretical Astroparticle Physics (TAsP-LNF). M.R. is partly supported by BG-NSF DN-08/14 from 14.12.2016.

Open Access. This article is distributed under the terms of the Creative Commons Attribution License (CC-BY 4.0), which permits any use, distribution and reproduction in any medium, provided the original author(s) and source are credited.

\section{References}

[1] F. Wilczek, Axions and Family Symmetry Breaking, Phys. Rev. Lett. 49 (1982) 1549 [INSPIRE].

[2] Y. Chikashige, R.N. Mohapatra and R.D. Peccei, Are There Real Goldstone Bosons Associated with Broken Lepton Number?, Phys. Lett. B 98 (1981) 265 [INSPIRE].

[3] G.B. Gelmini and M. Roncadelli, Left-Handed Neutrino Mass Scale and Spontaneously Broken Lepton Number, Phys. Lett. B 99 (1981) 411 [INSPIRE].

[4] R.D. Peccei and H.R. Quinn, CP Conservation in the Presence of Instantons, Phys. Rev. Lett. 38 (1977) 1440 [inSPIRE].

[5] R.D. Peccei and H.R. Quinn, Constraints Imposed by CP Conservation in the Presence of Instantons, Phys. Rev. D 16 (1977) 1791 [INSPIRE].

[6] S. Weinberg, A New Light Boson?, Phys. Rev. Lett. 40 (1978) 223 [inSPIRE].

[7] F. Wilczek, Problem of Strong $P$ and T Invariance in the Presence of Instantons, Phys. Rev. Lett. 40 (1978) 279 [INSPIRE].

[8] A. Arvanitaki, S. Dimopoulos, S. Dubovsky, N. Kaloper and J. March-Russell, String Axiverse, Phys. Rev. D 81 (2010) 123530 [arXiv:0905.4720] [INSPIRE].

[9] M. Cicoli, M. Goodsell and A. Ringwald, The type IIB string axiverse and its low-energy phenomenology, JHEP 10 (2012) 146 [arXiv:1206.0819] [INSPIRE].

[10] A. Hook, Anomalous solutions to the strong CP problem, Phys. Rev. Lett. 114 (2015) 141801 [arXiv: 1411.3325] [INSPIRE].

[11] H. Fukuda, K. Harigaya, M. Ibe and T.T. Yanagida, Model of visible QCD axion, Phys. Rev. D 92 (2015) 015021 [arXiv: 1504.06084] [INSPIRE].

[12] P.W. Graham, D.E. Kaplan and S. Rajendran, Cosmological Relaxation of the Electroweak Scale, Phys. Rev. Lett. 115 (2015) 221801 [arXiv:1504.07551] [InSPIRE].

[13] M. Bauer, M. Neubert and A. Thamm, Collider Probes of Axion-Like Particles, JHEP 12 (2017) 044 [arXiv: 1708.00443] [InSPIRE]. 
[14] M.J. Dolan, T. Ferber, C. Hearty, F. Kahlhoefer and K. Schmidt-Hoberg, Revised constraints and Belle II sensitivity for visible and invisible axion-like particles, JHEP 12 (2017) 094 [Erratum ibid. 03 (2021) 190] [arXiv:1709.00009] [INSPIRE].

[15] M. Bauer, M. Heiles, M. Neubert and A. Thamm, Axion-Like Particles at Future Colliders, Eur. Phys. J. C 79 (2019) 74 [arXiv:1808.10323] [inSPIRE].

[16] J.L. Feng, I. Galon, F. Kling and S. Trojanowski, Axionlike particles at FASER: The LHC as a photon beam dump, Phys. Rev. D 98 (2018) 055021 [arXiv:1806.02348] [INSPIRE].

[17] L. Harland-Lang, J. Jaeckel and M. Spannowsky, A fresh look at ALP searches in fixed target experiments, Phys. Lett. B 793 (2019) 281 [arXiv:1902.04878] [INSPIRE].

[18] D. Aloni, C. Fanelli, Y. Soreq and M. Williams, Photoproduction of Axionlike Particles, Phys. Rev. Lett. 123 (2019) 071801 [arXiv:1903.03586] [INSPIRE].

[19] B. Döbrich, J. Jaeckel and T. Spadaro, Light in the beam dump - ALP production from decay photons in proton beam-dumps, JHEP 05 (2019) 213 [Erratum ibid. 10 (2020) 046] [arXiv: 1904.02091] [INSPIRE].

[20] F. Ertas and F. Kahlhoefer, On the interplay between astrophysical and laboratory probes of MeV-scale axion-like particles, JHEP 07 (2020) 050 [arXiv:2004.01193] [INSPIRE].

[21] Y. Sakaki and D. Ueda, Searching for new light particles at the international linear collider main beam dump, Phys. Rev. D 103 (2021) 035024 [arXiv: 2009.13790] [INSPIRE].

[22] V. Brdar et al., Axion-like Particles at Future Neutrino Experiments: Closing the "Cosmological Triangle", Phys. Rev. Lett. 126 (2021) 201801 [arXiv:2011.07054] [INSPIRE].

[23] D.V. Kirpichnikov, V.E. Lyubovitskij and A.S. Zhevlakov, Implication of hidden sub-GeV bosons for the $(g-2)_{\mu},{ }^{8} B e-{ }^{4} H e$ anomaly, proton charge radius, EDM of fermions, and dark axion portal, Phys. Rev. D 102 (2020) 095024 [arXiv:2002.07496] [INSPIRE].

[24] D. Chang, W.-F. Chang, C.-H. Chou and W.-Y. Keung, Large two loop contributions to g- 2 from a generic pseudoscalar boson, Phys. Rev. D 63 (2001) 091301 [hep-ph/0009292] [INSPIRE].

[25] W.J. Marciano, A. Masiero, P. Paradisi and M. Passera, Contributions of axionlike particles to lepton dipole moments, Phys. Rev. D 94 (2016) 115033 [arXiv:1607.01022] [INSPIRE].

[26] J. Liu, C.E.M. Wagner and X.-P. Wang, A light complex scalar for the electron and muon anomalous magnetic moments, JHEP 03 (2019) 008 [arXiv: 1810.11028] [INSPIRE].

[27] F. Abu-Ajamieh, Probing Scalar and Pseudoscalar Solutions of the g-2 Anomaly, Adv. High Energy Phys. 2020 (2020) 1751534 [arXiv: 1810.08891] [INSPIRE].

[28] M. Bauer, M. Neubert, S. Renner, M. Schnubel and A. Thamm, Axionlike Particles, Lepton-Flavor Violation, and a New Explanation of $a_{\mu}$ and $a_{e}$, Phys. Rev. Lett. 124 (2020) 211803 [arXiv: 1908.00008] [INSPIRE].

[29] C. Cornella, P. Paradisi and O. Sumensari, Hunting for ALPs with Lepton Flavor Violation, JHEP 01 (2020) 158 [arXiv: 1911.06279] [inSPIRE].

[30] D.V. Kirpichnikov, V.E. Lyubovitskij and A.S. Zhevlakov, Constraints on CP-odd ALP couplings from EDM limits of fermions, Particles 3 (2020) 719 [arXiv: 2004.13656] [INSPIRE]. 
[31] A.J. Krasznahorkay et al., Observation of Anomalous Internal Pair Creation in ${ }^{8} B e: A$ Possible Indication of a Light, Neutral Boson, Phys. Rev. Lett. 116 (2016) 042501 [arXiv: 1504.01527] [INSPIRE].

[32] A. Krasznahorkay et al., New experimental results for the $17 \mathrm{MeV}$ particle created in ${ }^{8} \mathrm{Be}$, EPJ Web Conf. 137 (2017) 08010.

[33] A.J. Krasznahorkay et al., New evidence supporting the existence of the hypothetic X17 particle, arXiv:1910.10459 [INSPIRE].

[34] D. Firak et al., Confirmation of the existence of the X17 particle, EPJ Web Conf. 232 (2020) 04005.

[35] U. Ellwanger and S. Moretti, Possible Explanation of the Electron Positron Anomaly at $17 \mathrm{MeV}$ in ${ }^{8}$ Be Transitions Through a Light Pseudoscalar, JHEP 11 (2016) 039 [arXiv: 1609.01669] [INSPIRE].

[36] J.L. Feng et al., Particle physics models for the $17 \mathrm{MeV}$ anomaly in beryllium nuclear decays, Phys. Rev. D 95 (2017) 035017 [arXiv:1608.03591] [InSPIRE].

[37] J. Hewett et al., Fundamental Physics at the Intensity Frontier, in proceedings of the 2011 workshop on Fundamental Physics at the Intensity Frontier, (2012) [arXiv:1205.2671] [INSPIRE].

[38] R. Essig et al., Working Group Report: New Light Weakly Coupled Particles, in 2013 Community Summer Study on the Future of U.S. Particle Physics: Snowmass on the Mississippi (CSS2013), Minneapolis, U.S.A., July 29 - August 6 (2013) [arXiv:1311.0029] [INSPIRE] [http://www.slac.stanford.edu/econf/C1307292/docs/IntensityFrontier/NewLight-17.pdf].

[39] B. Batell, M. Pospelov and A. Ritz, Exploring Portals to a Hidden Sector Through Fixed Targets, Phys. Rev. D 80 (2009) 095024 [arXiv:0906.5614] [INSPIRE].

[40] DELPHI collaboration, Search for one large extra dimension with the DELPHI detector at LEP, Eur. Phys. J. C 60 (2009) 17 [arXiv:0901.4486] [InSPIRE].

[41] BaBAr collaboration, Search for Invisible Decays of a Dark Photon Produced in $e^{+} e^{-}$ Collisions at BaBar, Phys. Rev. Lett. 119 (2017) 131804 [arXiv:1702.03327] [INSPIRE].

[42] NA64 collaboration, Search for Axionlike and Scalar Particles with the NA64 Experiment, Phys. Rev. Lett. 125 (2020) 081801 [arXiv:2005.02710] [INSPIRE].

[43] BeLLe-II collaboration, The Belle II Physics Book, PTEP 2019 (2019) 123C01 [Erratum ibid. 2020 (2020) 029201] [arXiv:1808.10567] [INSPIRE].

[44] J.D. Bjorken et al., Search for Neutral Metastable Penetrating Particles Produced in the SLAC Beam Dump, Phys. Rev. D 38 (1988) 3375 [InSPIRE].

[45] M. Raggi and V. Kozhuharov, Proposal to Search for a Dark Photon in Positron on Target Collisions at DAФNE Linac, Adv. High Energy Phys. 2014 (2014) 959802 [arXiv: 1403.3041] [INSPIRE].

[46] M. Raggi, V. Kozhuharov and P. Valente, The PADME experiment at LNF, EPJ Web Conf. 96 (2015) 01025 [arXiv:1501.01867] [InSPIRE].

[47] P. Valente, POSEYDON - Converting the DAФNE Collider into a double Positron Facility: a High Duty-Cycle pulse stretcher and a storage ring, arXiv:1711.06877 [INSPIRE]. 
[48] P.F. Depta, M. Hufnagel and K. Schmidt-Hoberg, Updated BBN constraints on electromagnetic decays of MeV-scale particles, JCAP 04 (2021) 011 [arXiv:2011.06519] [INSPIRE].

[49] Y. Nomura and J. Thaler, Dark Matter through the Axion Portal, Phys. Rev. D 79 (2009) 075008 [arXiv: 0810.5397] [INSPIRE].

[50] M.J. Dolan, F. Kahlhoefer, C. McCabe and K. Schmidt-Hoberg, A taste of dark matter: Flavour constraints on pseudoscalar mediators, JHEP 03 (2015) 171 [Erratum ibid. 07 (2015) 103] [arXiv: 1412.5174] [INSPIRE].

[51] T.R. Slatyer, Indirect dark matter signatures in the cosmic dark ages. I. Generalizing the bound on s-wave dark matter annihilation from Planck results, Phys. Rev. D 93 (2016) 023527 [arXiv: 1506.03811] [INSPIRE].

[52] Planck collaboration, Planck 2018 results. VI. Cosmological parameters, Astron. Astrophys. 641 (2020) A6 [arXiv:1807.06209] [INSPIRE].

[53] Fermi-LAT collaboration, Updated search for spectral lines from Galactic dark matter interactions with pass 8 data from the Fermi Large Area Telescope, Phys. Rev. D 91 (2015) 122002 [arXiv: 1506.00013] [INSPIRE].

[54] M. Pospelov, A. Ritz and M.B. Voloshin, Secluded WIMP Dark Matter, Phys. Lett. B 662 (2008) 53 [arXiv:0711.4866] [inSPIRE].

[55] L. Darmé, S. Rao and L. Roszkowski, Signatures of dark Higgs boson in light fermionic dark matter scenarios, JHEP 12 (2018) 014 [arXiv:1807.10314] [INSPIRE].

[56] L. Darmé, S. Rao and L. Roszkowski, Light dark Higgs boson in minimal sub-GeV dark matter scenarios, JHEP 03 (2018) 084 [arXiv:1710.08430] [INSPIRE].

[57] G. Krnjaic, Probing Light Thermal Dark-Matter With a Higgs Portal Mediator, Phys. Rev. D 94 (2016) 073009 [arXiv: 1512.04119] [INSPIRE].

[58] J.A. Evans, A. Ghalsasi, S. Gori, M. Tammaro and J. Zupan, Light Dark Matter from Entropy Dilution, JHEP 02 (2020) 151 [arXiv:1910.06319] [INSPIRE].

[59] S. Dimopoulos, R. Esmailzadeh, L.J. Hall and N. Tetradis, Electroweak phase transition and dark matter abundance, Phys. Lett. B 247 (1990) 601 [INSPIRE].

[60] T. Cohen, D.E. Morrissey and A. Pierce, Changes in Dark Matter Properties After Freeze-Out, Phys. Rev. D 78 (2008) 111701 [arXiv:0808.3994] [InSPIRE].

[61] M. Millea, L. Knox and B. Fields, New Bounds for Axions and Axion-Like Particles with keV-GeV Masses, Phys. Rev. D 92 (2015) 023010 [arXiv:1501.04097] [InSPIRE].

[62] P.F. Depta, M. Hufnagel, K. Schmidt-Hoberg and S. Wild, BBN constraints on the annihilation of MeV-scale dark matter, JCAP 04 (2019) 029 [arXiv: 1901. 06944] [INSPIRE].

[63] P.F. Depta, M. Hufnagel and K. Schmidt-Hoberg, Robust cosmological constraints on axion-like particles, JCAP 05 (2020) 009 [arXiv: 2002.08370] [INSPIRE].

[64] J. Jaeckel, P.C. Malta and J. Redondo, Decay photons from the axionlike particles burst of type-II supernovae, Phys. Rev. D 98 (2018) 055032 [arXiv: 1702.02964] [INSPIRE].

[65] G.G. Raffelt, Astrophysical axion bounds, Lect. Notes Phys. 741 (2008) 51 [hep-ph/0611350] [INSPIRE]. 
[66] G. Lucente, P. Carenza, T. Fischer, M. Giannotti and A. Mirizzi, Heavy axion-like particles and core-collapse supernovae: constraints and impact on the explosion mechanism, JCAP 12 (2020) 008 [arXiv: 2008.04918] [inSPIRE].

[67] J.H. Chang, R. Essig and S.D. McDermott, Supernova 1987A Constraints on Sub-GeV Dark Sectors, Millicharged Particles, the QCD Axion, and an Axion-like Particle, JHEP 09 (2018) 051 [arXiv: 1803.00993] [INSPIRE].

[68] W. DeRocco, P.W. Graham, D. Kasen, G. Marques-Tavares and S. Rajendran, Supernova signals of light dark matter, Phys. Rev. D 100 (2019) 075018 [arXiv:1905.09284] [INSPIRE].

[69] F. D'Eramo, N. Fernandez and S. Profumo, Dark Matter Freeze-in Production in Fast-Expanding Universes, JCAP 02 (2018) 046 [arXiv:1712.07453] [INSPIRE].

[70] A. Sung, H. Tu and M.-R. Wu, New constraint from supernova explosions on light particles beyond the Standard Model, Phys. Rev. D 99 (2019) 121305 [arXiv:1903.07923] [InSPIRE].

[71] S.J. Brodsky, E. Mottola, I.J. Muzinich and M. Soldate, Laser induced Axion photoproduction, Phys. Rev. Lett. 56 (1986) 1763 [Erratum ibid. 57 (1986) 502] [INSPIRE].

[72] J.B. Dent et al., New Directions for Axion Searches via Scattering at Reactor Neutrino Experiments, Phys. Rev. Lett. 124 (2020) 211804 [arXiv: 1912.05733] [INSPIRE].

[73] E. Nardi, C.D.R. Carvajal, A. Ghoshal, D. Meloni and M. Raggi, Resonant production of dark photons in positron beam dump experiments, Phys. Rev. D 97 (2018) 095004 [arXiv: 1802.04756] [INSPIRE].

[74] L. Marsicano et al., Novel Way to Search for Light Dark Matter in Lepton Beam-Dump Experiments, Phys. Rev. Lett. 121 (2018) 041802 [arXiv:1807.05884] [INSPIRE].

[75] L. Marsicano et al., Dark photon production through positron annihilation in beam-dump experiments, Phys. Rev. D 98 (2018) 015031 [arXiv:1802.03794] [InSPIRE].

[76] Y.-S. Tsai, Pair Production and Bremsstrahlung of Charged Leptons, Rev. Mod. Phys. 46 (1974) 815 [Erratum ibid. 49 (1977) 421] [INSPIRE].

[77] J.D. Bjorken, R. Essig, P. Schuster and N. Toro, New Fixed-Target Experiments to Search for Dark Gauge Forces, Phys. Rev. D 80 (2009) 075018 [arXiv: 0906.0580] [InSPIRE].

[78] K. Jodłowski, F. Kling, L. Roszkowski and S. Trojanowski, Extending the reach of FASER, MATHUSLA, and SHiP towards smaller lifetimes using secondary particle production, Phys. Rev. D 101 (2020) 095020 [arXiv:1911.11346] [INSPIRE].

[79] L.I. Schiff, Energy-Angle Distribution of Thin Target Bremsstrahlung, Phys. Rev. 83 (1951) 252 [INSPIRE].

[80] A. Celentano, L. Darmé, L. Marsicano and E. Nardi, New production channels for light dark matter in hadronic showers, Phys. Rev. D 102 (2020) 075026 [arXiv:2006.09419] [INSPIRE].

[81] Y.-S. Tsai and V. Whitis, Thick target bremsstrahlung and target consideration for secondary particle production by electrons, Phys. Rev. 149 (1966) 1248 [INSPIRE].

[82] Y.-S. Tsai, Axion bremsstrahlung by an electron beam, Phys. Rev. D 34 (1986) 1326 [INSPIRE].

[83] J. Jaeckel and M. Spannowsky, Probing $\mathrm{MeV}$ to $90 \mathrm{GeV}$ axion-like particles with LEP and LHC, Phys. Lett. B 753 (2016) 482 [arXiv: 1509.00476] [INSPIRE]. 
[84] P.J. Fox, R. Harnik, J. Kopp and Y. Tsai, LEP Shines Light on Dark Matter, Phys. Rev. D 84 (2011) 014028 [arXiv: 1103.0240] [INSPIRE].

[85] A. Belyaev, N.D. Christensen and A. Pukhov, CalcHEP 3.4 for collider physics within and beyond the Standard Model, Comput. Phys. Commun. 184 (2013) 1729 [arXiv:1207.6082] [INSPIRE].

[86] DELPHI collaboration, Photon events with missing energy in $e^{+} e^{-}$collisions at $\sqrt{s}=130 \mathrm{GeV}$ to $209 \mathrm{GeV}$, Eur. Phys. J. C 38 (2005) 395 [hep-ex/0406019] [InSPIRE].

[87] BABAR collaboration, Search for Production of Invisible Final States in Single-Photon Decays of $\Upsilon(1 S)$, Phys. Rev. Lett. 107 (2011) 021804 [arXiv:1007.4646] [INSPIRE].

[88] R. Brun and F. Rademakers, ROOT: An object oriented data analysis framework, Nucl. Instrum. Meth. A 389 (1997) 81 [INSPIRE].

[89] E.M. Riordan et al., A Search for Short Lived Axions in an Electron Beam Dump Experiment, Phys. Rev. Lett. 59 (1987) 755 [inSPIRE].

[90] B. Batell, R. Essig and Z. Surujon, Strong Constraints on Sub-GeV Dark Sectors from SLAC Beam Dump E137, Phys. Rev. Lett. 113 (2014) 171802 [arXiv:1406. 2698] [inSPIRE].

[91] R.R. Dusaev, D.V. Kirpichnikov and M.M. Kirsanov, Photoproduction of axionlike particles in the NA64 experiment, Phys. Rev. D 102 (2020) 055018 [arXiv:2004.04469] [INSPIRE].

[92] PADME collaboration, First results on the performance of the PADME electromagnetic calorimeter, 2020 JINST 15 C05008 [arXiv:2002.11671] [INSPIRE].

[93] Muon G-2 collaboration, Final Report of the Muon E821 Anomalous Magnetic Moment Measurement at BNL, Phys. Rev. D 73 (2006) 072003 [hep-ex/0602035] [InSPIRE].

[94] T. Aoyama et al., The anomalous magnetic moment of the muon in the Standard Model, Phys. Rept. 887 (2020) 1 [arXiv: 2006. 04822] [INSPIRE].

[95] R.H. Parker, C. Yu, W. Zhong, B. Estey and H. Müller, Measurement of the fine-structure constant as a test of the Standard Model, Science 360 (2018) 191 [arXiv:1812.04130] [INSPIRE].

[96] T. Aoyama, T. Kinoshita and M. Nio, Revised and Improved Value of the QED Tenth-Order Electron Anomalous Magnetic Moment, Phys. Rev. D 97 (2018) 036001 [arXiv: 1712.06060] [INSPIRE].

[97] J.P. Leveille, The Second Order Weak Correction to $(G-2)$ of the Muon in Arbitrary Gauge Models, Nucl. Phys. B 137 (1978) 63 [INSPIRE].

[98] L. Darmé, L. Di Luzio, M. Giannotti and E. Nardi, Selective enhancement of the QCD axion couplings, Phys. Rev. D 103 (2021) 015034 [arXiv:2010.15846] [INSPIRE].

[99] F. Björkeroth, E.J. Chun and S.F. King, Flavourful Axion Phenomenology, JHEP 08 (2018) 117 [arXiv: 1806.00660] [INSPIRE].

[100] L. Morel, Z. Yao, P. Cladé and S. Guellati-Khélifa, Determination of the fine-structure constant with an accuracy of 81 parts per trillion, Nature 588 (2020) 61 [INSPIRE].

[101] L. Marsicano, M. Battaglieri, A. Celentano, R. De Vita and Y.-M. Zhong, Probing Leptophilic Dark Sectors at Electron Beam-Dump Facilities, Phys. Rev. D 98 (2018) 115022 [arXiv: 1812.03829] [INSPIRE].

[102] C.-Y. Chen, J. Kozaczuk and Y.-M. Zhong, Exploring leptophilic dark matter with NA64- $\mu$, JHEP 10 (2018) 154 [arXiv:1807.03790] [InSPIRE]. 
[103] Y. Kahn, G. Krnjaic, N. Tran and A. Whitbeck, $M^{3}$ : a new muon missing momentum experiment to probe $(g-2)_{\mu}$ and dark matter at Fermilab, JHEP 09 (2018) 153 [arXiv: 1804.03144] [INSPIRE].

[104] Belle-II collaboration, Search for an Invisibly Decaying $Z^{\prime}$ Boson at Belle II in $e^{+} e^{-} \rightarrow \mu^{+} \mu^{-}\left(e^{ \pm} \mu^{\mp}\right)$ Plus Missing Energy Final States, Phys. Rev. Lett. 124 (2020) 141801 [arXiv: 1912.11276] [INSPIRE].

[105] Muon G-2 collaboration, Measurement of the Positive Muon Anomalous Magnetic Moment to 0.46 ppm, Phys. Rev. Lett. 126 (2021) 141801 [arXiv:2104.03281] [INSPIRE]. 